\title{
Corticosterone Attenuates Reward-Seeking Behavior and Increases Anxiety via D2 Receptor Signaling in Ventral Tegmental Area Dopamine Neurons
}

\author{
${ }^{(D B}$ Beibei Peng, ${ }^{1,3}$ Qikuan Xu, ${ }^{1,3}$ Jing Liu, ${ }^{1,3}$ Sophie Guo, ${ }^{4}$ Stephanie L. Borgland, ${ }^{4}$ and Shuai Liu ${ }^{1,2,3}$ \\ ${ }^{1}$ Key Laboratory of Brain Functional Genomics (MOE\&STCSM), Affiliated Mental Health Center (ECNU), School of Psychology and Cognitive \\ Science, East China Normal University, Shanghai, 200062, China, ${ }^{2}$ Shanghai Changning Mental Health Center, Shanghai, 200335, China, ${ }^{3} \mathrm{NYU}$ - \\ ECNU Institute of Brain and Cognitive Science at NYU Shanghai, Shanghai, 200062, China, and ${ }^{4}$ Department of Physiology \& Pharmacology, \\ Hotchkiss Brain Institute, Cumming School of medicine, University of Calgary, Calgary, Alberta T2N 4N1, Canada
}

Corticosteroids (CORT) have been widely used in anti-inflammatory medication. Chronic CORT treatment can cause mesocorticolimbic system dysfunctions, which are known to play a key role for the development of psychiatric disorders. The VTA is a critical site in the mesocorticolimbic pathway and is responsible for motivation and reward-seeking behaviors. However, the mechanism by which chronic CORT alters VTA dopamine neuronal activity is largely unknown. We treated periadolescent male mice with vehicle, $1 \mathrm{~d}$, or $7 \mathrm{~d}$ CORT in the drinking water, examined behavioral impacts with light/dark box, elevated plus maze, operant chamber, and open field tests, measured the effects of CORT on VTA dopamine neuronal activity using patch-clamp electrophysiology and dopamine concentration using fast-scan cyclic voltammetry, and tested the effects of dopamine D2 receptor (D2R) blockade by intra-VTA infusion of a D2R antagonist. CORT treatment induced anxiety-like behavior as well as decreased food-seeking behaviors. We show that chronic CORT treatment decreased excitability and excitatory synaptic transmission onto VTA dopamine neurons. Furthermore, chronic CORT increased somatodendritic dopamine concentration. The D2R antagonist sulpiride restored decreased excitatory transmission and excitability of VTA dopamine neurons. Furthermore, sulpiride decreased anxiety-like behavior and rescued food-seeking behavior in mice with chronic CORT exposure. Together, $7 \mathrm{~d}$ CORT treatment induces anxiety-like behavior and impairs food-seeking in a mildly aversive environment. D2R signaling in the VTA might be a potential target to ameliorate chronic CORT-induced anxiety and reward-seeking deficits.

Key words: anxiety; corticosterone; D2 receptor; dopamine; reward; ventral tegmental area

\section{Significance Statement}

With widespread anti-inflammatory effects throughout the body, corticosteroids (CORT) have been used in a variety of therapeutic conditions. However, long-term CORT treatment causes cognitive impairments and neuropsychiatric disorders. The impact of chronic CORT on the mesolimbic system has not been elucidated. Here, we demonstrate that $7 \mathrm{~d}$ CORT treatment increases anxiety-like behavior and attenuates food-seeking behavior in a mildly aversive environment. By elevating local dopamine concentration in the VTA, a region important for driving motivated behavior, CORT treatment suppresses excitability and synaptic transmission onto VTA dopamine neurons. Intriguingly, blockade of D2 receptor signaling in the VTA restores neuronal excitability and food-seeking and alleviates anxiety-like behaviors. Our findings provide a potential therapeutic target for CORT-induced reward deficits.

Received Sep. 26, 2020; revised Dec. 8, 2020; accepted Dec. 14, 2020.

Author contributions: B.P., Q.X., J.L., S.G., and S.L. performed research; B.P., Q.X., J.L., S.G., and S.L. analyzed data; B.P. wrote the first draft of the paper; S.L.B. and S.L. designed research; S.L.B. edited the paper; S.L. wrote the paper.

This work was supported by National Natural Science Foundation of China 31800856, Shanghai Pujiang Program 18PJ1402600, Key Specialist Projects of Shanghai Municipal Commission of Health and Family Planning ZK2015B01, and Programs Foundation of Shanghai Municipal Commission of Health and Family Planning 201540114. S.L. was supported by NYU-ECNU Institute of Brain and Cognitive Science at NYU Shanghai. S.L.B. was supported by a Tier I Canada Research Chair and Canadian Institutes of Health
Research Operating Grant FDN-147473. This work was also supported by the Hotchkiss Brain Institute (HBI's) fund for depression research to S.L.B.

The authors declare no competing financial interests.

Correspondence should be addressed to Shuai Liu at sliu@psy.ecnu.edu.cn.

https://doi.org/10.1523/JNEUROSCI.2533-20.2020

Copyright $\odot 2020$ Peng et al.

This is an open-access article distributed under the terms of the Creative Commons Attribution 4.0 International license, which permits unrestricted use, distribution and reproduction in any medium provided that the original work is properly attributed. 


\section{Introduction}

Corticosteroids (CORT) have been used for a variety of conditions, such as pneumonia, asthma, severe allergies, and arthritis, because of its widespread anti-inflammatory effects (Rhen and Cidlowski, 2005). However, long-term exposure to CORT medications may cause side effects, including cognitive impairments and neuropsychiatric disorders (Sonino and Fava, 2001; Coluccia et al., 2008; Pivonello et al., 2015; Yasir and Sonthalia, 2019). While the physiological effects of acute CORT in the brain are well documented (Mitra and Sapolsky, 2008; Kim et al., 2014; Myers et al., 2014; Joels, 2018), the impact of longer-term CORT treatment, consistent with systemic anti-inflammatory treatment, on the mesolimbic system has not been elucidated.

VTA dopamine neurons play an important role in learning the incentive value of stimuli or actions to guide motivated behavior and thus play key roles in addiction and mood disorders. These neurons are highly sensitive to corticotropin releasing factor and stress (for review, see Polter and Kauer, 2014; Hollon et al., 2015), yet less is known about how they respond to glucocorticoids. CORT activates glucocorticoid receptors (GRs) or mineralocorticoid receptors (MRs), and these receptors are subsequently translocated to the nucleus and act as transcription factors (McEwen et al., 1986). However, rapid nongenomic cellular mechanisms have been observed on activation of GRs (Tasker and Herman, 2011).

Several lines of evidence indicate that the midbrain dopamine system could be implicated in impairments associated with chronic CORT treatment. Both MRs and GRs are expressed in a subset of VTA dopamine neurons (Harfstrand et al., 1986; Ronken et al., 1994; Hensleigh and Pritchard, 2013). Activation of GRs by either in vivo injection or acute incubation of VTA slices with dexamethasone potentiates synaptic strength onto dopamine neurons (Daftary et al., 2009). Furthermore, stressinduced plasticity in the VTA is blocked by administration of a GR antagonist (Saal et al., 2003). Acute CORT application potentiated NMDA currents in VTA slices (Cho and Little, 1999) and NMDA neurotoxicity of VTA neurons in organotypic VTA and NAc cocultures (Berry et al., 2016). Because major depressive disorder and reduced motivation are frequently reported in patients with CORT treatment-induced Cushing syndrome (Wagenmakers et al., 2012; Pivonello et al., 2015), chronic CORT treatment may dysregulate dopamine signaling to influence behavior. However, little is known about how chronic CORT treatment influences synaptic transmission in the VTA. In the present study, we tested the hypothesis that chronic CORT exposure impacts VTA neurophysiology and reward-seeking behaviors using an oral CORT treatment mouse model (Gourley et al., 2008; Kinlein et al., 2017).

\section{Materials and Methods}

\section{Animals and CORT treatment}

All protocols were in accordance with the ethical guidelines established by the Canadian Council for Animal Care and were approved by the University of Calgary Animal Care Committee and by the East China Normal University Animal Care Committee. P21 male C57BL/6 mice obtained from Charles River Laboratories or The Jackson Laboratory were housed in a $12 \mathrm{~h}$ light/12 h dark cycle in a temperature- and humidity-controlled environment with food and water freely available. All efforts were made to minimize animal suffering and reduce the number of animals used. Oral CORT treatment was conducted by replacing drinking water with either $100 \mu \mathrm{g} / \mathrm{ml}$ CORT dissolved in ethanol $(1 \%$ ethanol final concentration) or vehicle (1\% ethanol solution) (Kinlein et al., 2017; Moda-Sava et al., 2019). Body weight, food consumption, and water intake were measured. To measure CORT level in mice, blood was collected at 3-5 h after light on and clotted at room temperature for $\sim 30 \mathrm{~min}$ followed by centrifugation $\left(955 \times g\right.$ for $30 \mathrm{~min}$ at $\left.4^{\circ} \mathrm{C}\right)$. The serum was stored at $-80^{\circ} \mathrm{C}$ before assaying. Serum was diluted 1:500 for corticosterone ELISA (Cayman Chemical, \#501320) following the assay instruction. Glucose tolerance test was performed using glucose meter (Roche Diagnostic). After overnight fasting, animals were weighed and basal glucose was measured. D-glucose was then injected $(2 \mathrm{~g} / \mathrm{kg}, 0.9 \%$ saline, i.p.), and tail blood was collected at 10, 20, 30, 45, 60, 90, 120, and $180 \mathrm{~min}$ and measured with a glucometer.

\section{Electrophysiology and electrochemistry}

Slice electrophysiology experiments were performed from 4- to 5-weekold male C57BL/6 mice. Mice were anesthetized with either isoflurane or $0.6 \%$ pentobarbital sodium, decapitated, and brains were extracted. VTA slices $(250 \mu \mathrm{m})$ were prepared with an ice-cold sucrose solution containing (in mM): $87 \mathrm{NaCl}, 2.5 \mathrm{KCl}, 1.25 \mathrm{NaH}_{2} \mathrm{PO}_{4}, 25 \mathrm{NaHCO}_{3}, 7 \mathrm{MgCl}_{2}, 0.5$ $\mathrm{CaCl}_{2}, 75$ sucrose, using a vibratome (Leica Microsystems). Slices were placed in a holding chamber and allowed to recover for at least $1 \mathrm{~h}$ before moving to the recording chamber and superfused with aCSF solution containing the following (in mM): $126 \mathrm{NaCl}, 1.6 \mathrm{KCl}, 1.1 \mathrm{NaH}_{2} \mathrm{PO}_{4}$, $1.4 \mathrm{MgCl}_{2}, 2.4 \mathrm{CaCl}_{2}, 26 \mathrm{NaHCO}_{3}$ and 11 glucose, saturated with $95 \%$ $\mathrm{O}_{2} / 5 \% \mathrm{CO}_{2}$ at $32^{\circ} \mathrm{C}$. Cells were visualized using infrared differential interference contrast video microscopy.

Whole-cell patch-clamp recordings were made using a MultiClamp 700B amplifier (Molecular Devices). Electrodes (3-5 M $\Omega$ ) contained the following (in mM): 117 cesium methanesulfonate, 20 HEPES, 0.4 EGTA, $2.8 \mathrm{NaCl}, 5 \mathrm{TEA}-\mathrm{Cl}, 2.5 \mathrm{Mg}$-ATP, and 0.25 Na-GTP (pH 7.2-7.3, 270$280 \mathrm{mOsm})$. Biocytin (0.2\%) was added to the internal solution before every experiment. Series resistance (10-25 $\mathrm{M} \Omega$ ) and input resistance were monitored online with a $10 \mathrm{mV}$ depolarizing step $(400 \mathrm{~ms})$ given before every afferent stimulus. Dopamine neurons were identified by the presence of a hyperpolarizing cation current, a predictor of lateral VTA dopamine neurons medial to the medial terminals of the optic nucleus in mice (Wanat et al., 2008) and were further confirmed by post hoc staining for the rate-limiting enzyme, TH (Fig. $1 A$ ).

A bipolar stimulating electrode was placed $100-300 \mu \mathrm{m}$ rostral to the recording electrode to stimulate excitatory afferents at $0.1 \mathrm{~Hz}$. AMPARmediated EPSCs were recorded in cells voltage-clamped at $-60 \mathrm{mV}$ in the presence of picrotoxin $(100 \mu \mathrm{M})$ to block $\mathrm{GABA}_{\mathrm{A}}$ receptor-mediated IPSCs. EPSCs were filtered at $2 \mathrm{kHz}$, digitized at $10 \mathrm{kHz}$, and collected using pClamp 10 (Molecular Devices). Currents traces were constructed by averaging 10 consecutive EPSCs. Paired pulses were evoked with a $50 \mathrm{~ms}$ interstimulus interval. To calculate the AMPAR/NMDAR ratio, an average of 12 EPSCs at $40 \mathrm{mV}$ was computed before and after application of the NMDAR blocker AP5 $(50 \mu \mathrm{M})$ for $5 \mathrm{~min}$. NMDAR responses were calculated by subtracting the average response in the presence of AP5 (AMPAR only) from that seen in its absence; the peak of the AMPAR EPSC was divided by the peak of the NMDAR EPSC to yield an AMPAR/NMDAR ratio.

To test excitatory and inhibitory transmission, and calculate their ratio, we recorded mEPSCs and mIPSCs from same neuron. Inhibitory and excitatory current reversal potentials were first tested and calculated from $I-V$ curve, respectively. mEPSCs were recorded in cells voltageclamped at $-68 \mathrm{mV}$ (inhibitory reversal potential) in TTX (500 nM). mIPSCs were voltage clamped at $10 \mathrm{mV}$ (excitatory reversal potential) in TTX (500 nM). mIPSCs and mEPSCs were analyzed using Mini60 Mini Analysis Program (Synaptosoft). Detection criteria were set at $>12 \mathrm{pA}$ (at least $2 \times$ rms noise), $<1.75 \mathrm{~ms}$ rise time and $<4 \mathrm{~ms}$ decay time for AMPAR mEPSCs, and for mIPSCs $>15 \mathrm{pA}$ (at least $2 \times$ rms noise), $<4$ ms rise time and $<10 \mathrm{~ms}$ decay time to account for the longer decay kinetics of $\mathrm{GABA}_{\mathrm{A}}$ receptors (Godfrey and Borgland, 2020).

To investigate neuronal excitability, glass electrodes (3-5 $\mathrm{m} \Omega$ ) contained the following (in mM): $140 \mathrm{~K} \mathrm{D}$-gluconate, $5 \mathrm{KCl}, 10 \mathrm{HEPES}, 0.2$ EGTA, $2 \mathrm{NaCl}, 2.5 \mathrm{Mg}$-ATP, and 0.25 Na-GTP (pH 7.2-7.3, 270-280 mOsm). Neurons were held at $-60 \mathrm{mV}$ and depolarizing current steps ( $400 \mathrm{~ms}$, from 0 to $350 \mathrm{mV}$ in $50 \mathrm{mV}$ steps) were applied in the absence of transmitter receptor blockers. The slope of the number of spikes per depolarizing step was used as a measure of excitability. 
A

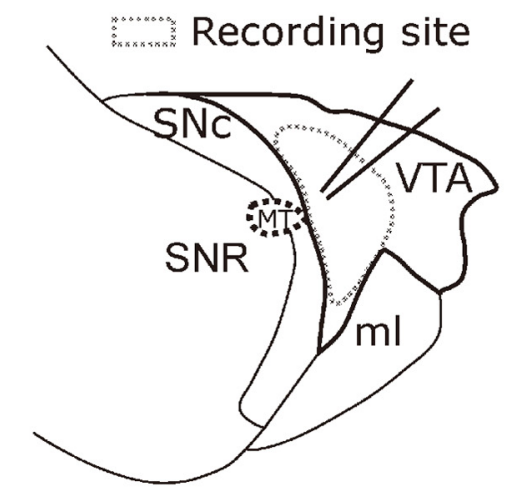

B

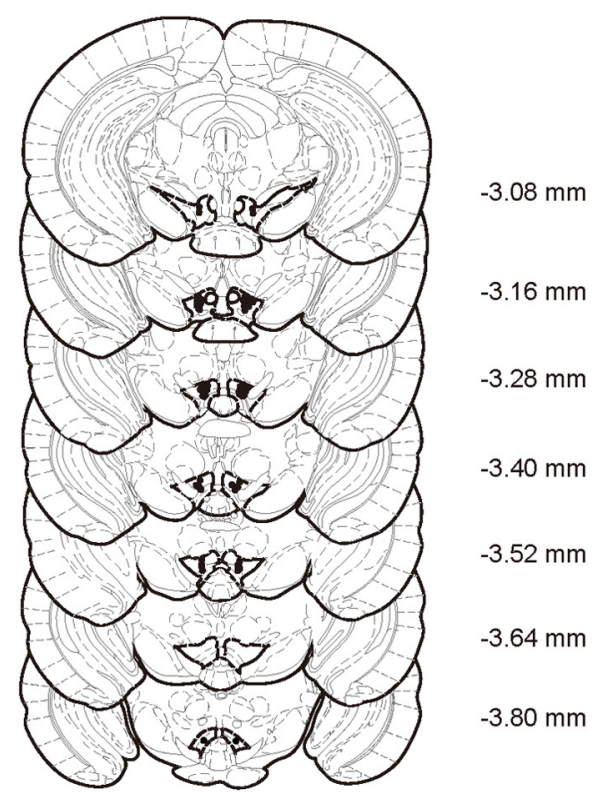

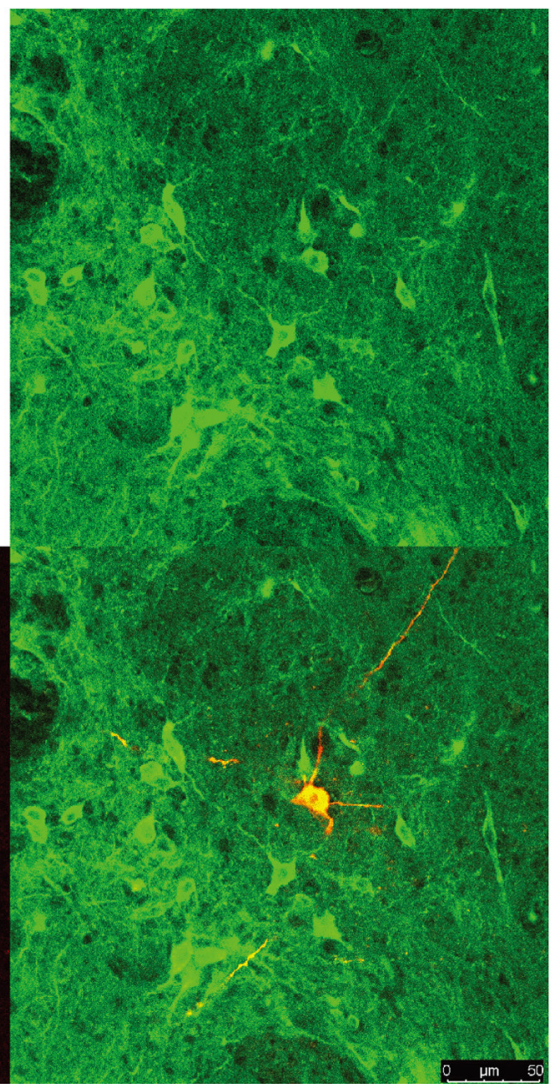

C

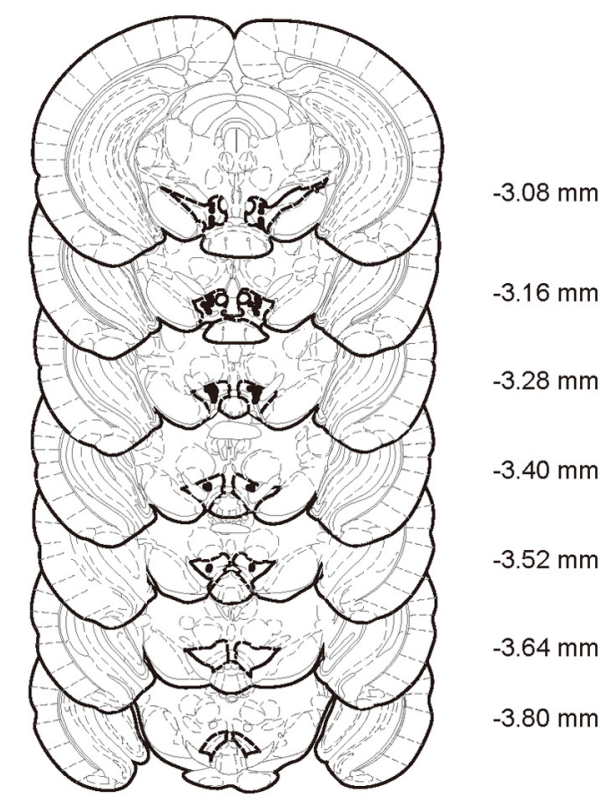

Figure 1. Schematic recording sites and reconstructed bilateral injection sites in the VTA. A, Schematic recording sites (top left). Example confocal image of biocytin-labeled TH-positive neuron. Post hoc TH immunofluorescence was identified in the VTA (top right). Biocytin was administered via the patch pipette during the electrophysiological recording (bottom left). Merged image of a biocytin-labeled TH-positive neuron (bottom right). Scale bar, $50 \mu \mathrm{m}$. B, Injection sites in the reward-seeking behavior test and $(\boldsymbol{C})$ injection sites in the OF, EPM, and PR tests are shown in coronal sections. Distance from bregma is shown to the right of each section (in millimeters).

Evoked extracellular dopamine $\left([\mathrm{DA}]_{\mathrm{o}}\right)$ was measured using fastscan cyclic voltammetry with carbon-fiber microelectrodes as previously reported (Mebel et al., 2012). Briefly, carbon fibers ( $7 \mu \mathrm{m}$ diameter; Goodfellow) were pulled in glass electrodes and cut to a final exposed length of $\sim 150 \mu \mathrm{m}$. Triangular waveforms (holding at $-0.4 \mathrm{~V}$ ) at $10 \mathrm{~Hz}$
( -0.4 to $1.0 \mathrm{~V}$ vs $\mathrm{Ag} / \mathrm{AgCl}$ at $400 \mathrm{~V} / \mathrm{s}$ scan rate) were used. Electrical stimulation $(40 \mathrm{~Hz}, 20$ pulses) was applied with a bipolar stimulating electrode positioned flush with the tissue for local surface stimulation. The voltammetric electrode was positioned between the tips with the aid of a binocular microscope, then lowered $50-100 \mu \mathrm{m}$ into the tissue. 
Dopamine was identified by characteristic oxidation and reduction peak potentials ( 600 and $-200 \mathrm{mV}$ vs $\mathrm{Ag} / \mathrm{AgCl}$ ). To determine the time course of dopamine, the current at the peak oxidation $(600 \mathrm{mV})$ was plotted against time. Calibration solutions were made from stock solutions in $0.1 \mathrm{M} \mathrm{HClO}_{4}$ immediately before use.

\section{Behavioral tests}

Reward-seeking behavior test. The reward-seeking behavior test was modified from light/dark box test (Teegarden and Bale, 2007), whereby mice were required to overcome their innate aversion to open light spaces to seek high-fat (HF) food. The apparatus was an acrylic box (45 $\mathrm{cm}$ length $\times 27 \mathrm{~cm}$ width $\times 27 \mathrm{~cm}$ height) divided into light and dark chambers. The light chamber $(27 \mathrm{~cm}$ length $\times 27 \mathrm{~cm}$ width $\times 27 \mathrm{~cm}$ height) was of white acrylic and was connected via an opening $(7.5 \times$ $7.5 \mathrm{~cm})$ at floor level to the black acrylic chamber $(18 \mathrm{~cm}$ length $\times 27 \mathrm{~cm}$ width $\times 27 \mathrm{~cm}$ height). A pellet of HF food (Research Diets, d12492) was placed in the center of the light chamber, and the area around food $(9 \mathrm{~cm} \times 9 \mathrm{~cm})$ was defined as food zone. Mice were pre-exposed to HF food briefly $2 \mathrm{~d}$ before the test to avoid neophobia. Mice were habituated to the test room before the test. A lamp was placed $2.5 \mathrm{~m}$ above the test chamber creating 92.6-108lux of light. Mice were placed in the light chamber facing the opening into the dark chamber, and Any-Maze software was used to record and track the mice during a $10 \mathrm{~min}$ trial. The apparatus was cleaned between each subject.

Elevated plus maze (EPM). The acrylic maze consists of 2 open arms and 2 closed arms $(30 \mathrm{~cm}$ in length and $5 \mathrm{~cm}$ in width, $15 \mathrm{~cm}$ wall for closed arm). Distance traveled and time spent in each arm during 10 min tests were recorded and analyzed by Any-Maze software. The apparatus was cleaned between each subject.

Open field test (OF). Tru scan system with an arena of $26 \mathrm{~cm}$ (length) $\times$ $26 \mathrm{~cm}$ (width) was used in the test. Mice were placed in the center of the arena. Distance traveled was recorded and analyzed automatically by the system during a $10 \mathrm{~min}$ trial. The apparatus was cleaned between each subject.

Progressive ratio test $(P R)$. Bussey-Saksida touchscreen chamber (Campden Instruments) was used in the PR test. During the training phase, mice were habituated to the chambers for 20 min with $200 \mu \mathrm{l}$ milkshake (Yazoo Strawberry UHT milkshake; Friesland Campina) delivered to the magazine on the first day. After habituation, mice were trained to perform the specific operant response (fixed ratio [FR] training, FR1, FR3, FR5) to get a reward ( $20 \mu \mathrm{l}$ milkshake) over several sessions ( 1 or 2 sessions per day). Criterion of a successful session was defined as completion of 30 trials in $60 \mathrm{~min}$. FR5 was conducted three consecutive sessions to ensure animals developed high selectivity for the target-illuminated screen location. Following FR5, mice progressed to three consecutive PR schedule sessions, in which a reward was delivered with a progressively increasing operant response requirement (i.e., 1, 5, 9,13 , etc.). During the training phase, mice were food-restricted $(\sim 85 \%$ of their daily food intake). After $7 \mathrm{~d}$ CORT treatment, three consecutive PR schedule tests were conducted. Task performance was evaluated by breakpoint, defined as the number of target responses emitted by an ani$\mathrm{mal}$ in the last successfully completed trial of a session.

Food preference test. Mice were tested for HF food preference using a two-bowl choice procedure. Each animal was housed individually during the $7 \mathrm{~d}$ test period. Animals were given two bowls, containing HF food and standard chow, respectively. Water was provided ad libitum. Every $24 \mathrm{~h}$, the amounts of HF food and lab chow consumed were recorded. To prevent potential location preference, the positions of the bowls were changed every $24 \mathrm{~h}$. The preference for the HF food was determined as the percentage of HF food ingested relative to the total food intake.

\section{Intra-VTA cannulations and microinjections}

Mice (4-5 weeks old) were anesthetized and placed in a stereotaxic frame (Kopf). Mice were implanted with bilateral guide cannulas (26 gauge; RWD 62064) into the VTA (anteroposterior, $-3.2 \mathrm{~mm}$; mediolateral, $\pm 0.5 \mathrm{~mm}$; dorsoventral, $-4.6 \mathrm{~mm}$ ). For micro-infusions, mice had two habituation sessions, by performing mock perfusions with a microinjector cut above the length of the cannula. On test days, micro-infusions were conducted by using 32-gauge microinjectors (RWD 62264) that protruded $0.2 \mathrm{~mm}$ below the base of the guide cannula to a final dorsoventral coordinate of $-4.8 \mathrm{~mm}$. Vehicle (aCSF) or D2 receptor (D2R) antagonist sulpiride $(0.15 \mu \mathrm{g} /$ side $)$ was infused bilaterally into the VTA $(0.2 \mu \mathrm{l}$ at $0.1 \mu \mathrm{l} / \mathrm{min})$, and microinjectors were left in place for $3 \mathrm{~min}$ after the injection. Mice were then returned to their home cage for $15 \mathrm{~min}$ before the behavioral assay. Injection placements were checked after the test (Fig. 1B,C).

\section{Drugs}

Corticosterone, picrotoxin, and ketoprofen were purchased from Cayman Chemical. FITC-AffiniPure Donkey Anti-Mouse IgG $(\mathrm{H}+\mathrm{L})$ and AlexaFluor-594-IgG Fraction Monoclonal Mouse Anti-Biotin were purchased from Jackson ImmunoResearch Laboratories. TTX was purchased from the Research Institute of the Aquatic Products of Hebei. All other regents and drugs were purchased from Sigma Millipore, Tocris Bioscience, or BBI Life Sciences.

\section{Data analysis}

All values are expressed as mean \pm SEM in addition to individual values. Statistical significance was assessed by using two-tailed Student's $t$ tests. Two-way ANOVA was used for multiple group comparisons unless otherwise indicated. $N$ refers to the number of cells recorded from $n$ mice. Data met the assumptions of equal variances and normality unless otherwise indicated. Excel (Microsoft) and Prism (GraphPad Prism 7 Software) were used to perform data processing and statistical analysis.

\section{Results}

\section{Impaired food-seeking and elevated anxiety in $7 \mathrm{~d}$ CORT- treated mice}

Chronic CORT treatment can induce metabolic dysfunction (Karatsoreos et al., 2010). Seven day CORT treatment significantly increased serum CORT level (vehicle: $21 \pm 2.0, n=8$; CORT: $56 \pm 14, n=8 ; t_{(14)}=2.5, p=0.024$; Fig. $2 A$ ). Baseline glucose level was significantly reduced in CORT mice after overnight fasting (vehicle: $6.2 \pm 0.5, n=8$; CORT: $3.7 \pm 0.4, n=6$; $t_{(12)}=3.5, p=0.0048$; Fig. $2 B$ ) with impaired glucose tolerance $\left(F_{(8,96)}=3.2, p=0.0027\right.$; Fig. $\left.2 C\right)$. Body weight gain was significantly decreased in $7 \mathrm{~d}$ CORT mice compared with vehicle mice (vehicle: $5.3 \pm 0.7 \mathrm{~g}, n=12$; CORT: $3.0 \pm 0.4 \mathrm{~g}, n=9 ; t_{(19)}=2.4$, $p=0.027$; Fig. $2 D$ ), while water and food intake increased (water: vehicle: $34 \pm 1 \mathrm{~g}, n=12$; CORT: $47 \pm 3 \mathrm{~g}, n=11 ; t_{(21)}=3.9$, $p=0.0008$; food: vehicle: $27 \pm 0.7 \mathrm{~g}, n=12$; CORT: $30 \pm 1 \mathrm{~g}$, $n=11 ; t_{(21)}=3.1, p=0.0055$; Fig. $\left.2 E, F\right)$. Mice acutely exposed to CORT $(1 \mathrm{~d})$ had increased water intake (vehicle: $5 \pm 0.2 \mathrm{~g}$, $n=11$; CORT: $6 \pm 0.5 \mathrm{~g}, n=10$; water intake $\times$ CORT interaction: $\left.F_{(1,40)}=11.16, p=0.0018\right)$, while weight gain and food intake were not significantly different in $1 \mathrm{~d}$ CORT mice. However, CORT treatment did not change preference for HF food when both chow and HF food were provided in home cage $\left(F_{(6,84)}=\right.$ $0.49, p=0.82$; Fig. $2 G$ ), suggesting reward sensitivity per se was not altered. Together, these results suggest $7 \mathrm{~d}$ CORT has significant metabolic effects on mice.

To test whether chronic CORT treatment influences foodseeking, we used a modified light dark box, whereby mice must enter the open light field to explore food (Fig. 3A) (Teegarden and Bale, 2007; Liu et al., 2016). Seven day CORT treatment significantly decreased food zone entries (vehicle: $46 \pm 6, n=6$; CORT: $18 \pm 5, n=6 ; t_{(10)}=3.5, p=0.0053$; Fig. $3 B$ ) and food zone time (vehicle: $117 \pm 22 \mathrm{~s}, n=6$; CORT: $24 \pm 11 \mathrm{~s}, n=6$; $t_{(10)}=3.8, p=0.0036$; Fig. $3 C$ ), with no change in light box distance traveled (vehicle: $10 \pm 0.9 \mathrm{~m}, n=6$; CORT: $7 \pm 0.9 \mathrm{~m}$, $n=6 ; t_{(10)}=1.9, p=0.079$; Fig. $3 D$ ). In addition, CORT treatment did not change total distance traveled of an open field (vehicle: $2120 \pm 151 \mathrm{~cm}, n=9 ;$ CORT: $2247 \pm 116 \mathrm{~cm}, n=8 ; t_{(15)}=0.65$, 

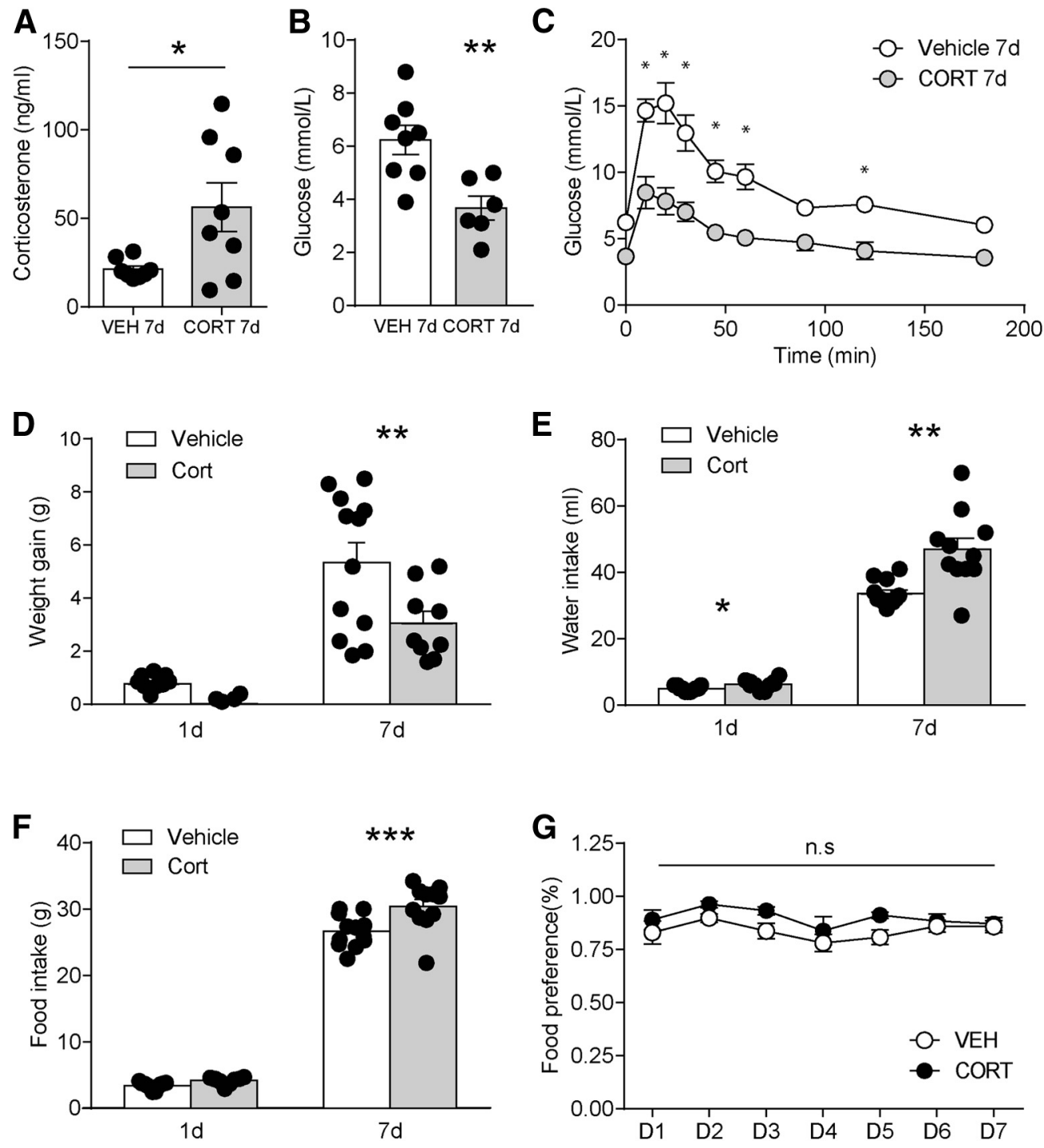

Figure 2. Metabolic changes in CORT-treated mice. A, Serum corticosterone level. B, Basal glucose level. C, Glucose tolerance curve. D, Weight gain. $\boldsymbol{E}$, Water intake. $\boldsymbol{F}$, Food intake. G, Food preference for $\mathrm{HF}$ diet compared with chow during the $7 \mathrm{~d}$ vehicle or CORT treatment. Error bars indicate SEM. $* p<0.05 . * * p<0.01$. $* * * p<0.001$. n.s., not significant.

$p=0.52$; Fig. $3 E$ ). Thus, decreased food-seeking was not because of altered locomotor activity. EPM test was used to test anxietylike behavior. Seven day CORT mice exhibited decreased open arm time (vehicle: $103 \pm 16 \mathrm{~s}, n=9$; CORT: $47 \pm 13 \mathrm{~s}, n=10$; $t_{(17)}=2.7, p=0.015$; Fig. $3 F$ ) and open arm entries (vehicle: $29 \pm 2, n=9$; CORT: $18 \pm 4, n=10 ; t_{(17)}=2.5, p=0.024$; Fig. $3 G$ ), with no difference in distance traveled (vehicle: $21 \pm 2$, $n=9$; CORT: $18 \pm 3, n=10 ; t_{(17)}=0.81, p=0.43$; Fig. $3 H$ ). These results are consistent with anxiety-like behaviors in CORT mice, which could contribute to impaired reward-seeking behaviors.

To test how long the $7 \mathrm{~d}$ CORT effects last, the same behavior paradigms were tested 2 weeks after CORT treatment in another group of mice. No significant change was found in food zone entries (vehicle: $19 \pm 4, n=10$; CORT: $18 \pm 3, n=10 ; t_{(18)}=0.34$, $p=0.74$; Fig. $4 A$ ), food zone time (vehicle: $19 \pm 4$ s, $n=10$; CORT: $32 \pm 5 \mathrm{~s}, n=10 ; t_{(18)}=2.0, p=0.057$; Fig. $4 B$ ), and light box distance traveled (vehicle: $8 \pm 1 \mathrm{~m}, n=10$; CORT: $8 \pm 1 \mathrm{~m}$, $n=10 ; t_{(18)}=0.14, p=0.89$; Fig. $4 C$ ) in the modified light-dark box test. Two weeks after $7 \mathrm{~d}$ CORT exposure, mice still displayed decreased open arm entries (vehicle: $29 \pm 2, n=10$; CORT: $24 \pm 1, n=10 ; t_{(18)}=2.5, p=0.020$; Fig. $4 E$ ) with no change in open arm time (vehicle: $140 \pm 15$ s, $n=10$; CORT:
$114 \pm 12 \mathrm{~s}, n=10 ; t_{(18)}=1.4, p=0.18$; Fig. $\left.4 D\right)$ and distance traveled (vehicle: $18 \pm 1 \mathrm{~m}, n=10$; CORT: $18 \pm 2 \mathrm{~m}, n=10 ; t_{(18)}=$ $0.12, p=0.91$; Fig. $4 F)$ in the EPM test. There was no significant difference in total distance (vehicle: $2587 \pm 82 \mathrm{~cm}, n=10$; CORT: $2352 \pm 213 \mathrm{~cm}, n=10 ; t_{(18)}=1.0, p=0.32$; Fig. $\left.4 G\right)$ of the OF test.

\section{Decreased VTA dopamine neuron excitability and} presynaptic glutamate release in $7 \mathrm{~d}$ CORT-treated mice

To examine the excitability of VTA dopamine neurons after CORT treatment, we measured the spike number elicited by depolarizing current steps. One day CORT did not alter the current evoked firing or excitability of dopamine neurons compared with vehicle: (current injection $\times 1 \mathrm{~d}$ CORT interaction: $F_{(7,160)}=0.3825, p=0.9116$, Fig. $5 A, B$; slope: vehicle: $1.0 \pm 0.06$ per $100 \mathrm{pA}(\mathrm{N} / n=11 / 3)$ vs $1 \mathrm{~d}$ CORT $1.1 \pm 0.07$ per $100 \mathrm{pA}$ $(N / n=11 / 4), t_{(20)}=1.7, p=0.11$, Fig. $\left.5 C\right)$. In contrast, dopamine neurons from $7 \mathrm{~d}$ CORT mice had reduced current-evoked firing and decreased excitability compared with vehicle treatment (current injection $\times 7 \mathrm{~d}$ CORT interaction: $F_{(7,119)}=3.147, p=$ 0.0044, Fig. $5 D, E$; slope: vehicle: $1.1 \pm 0.1$ per $100 \mathrm{pA}(N / n=10 / 4)$; 
A<smiles>CC1CC2CC2CC2CC1C2</smiles>

Vehicle or CORT<smiles>C1=CCCC1</smiles>

$11213 / 4|5| 6 \mid 7$

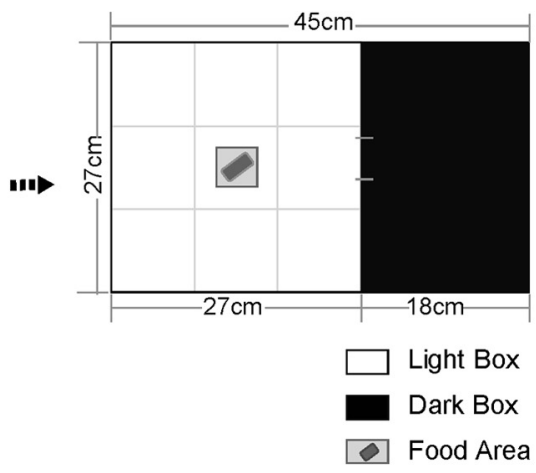

D

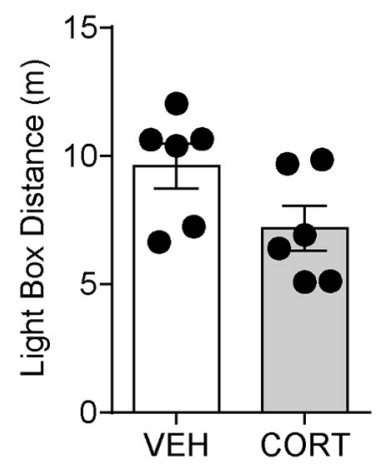

G

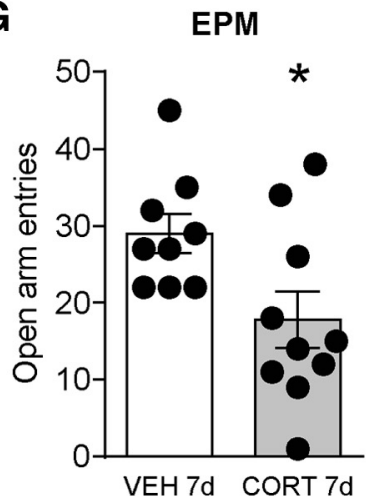

B

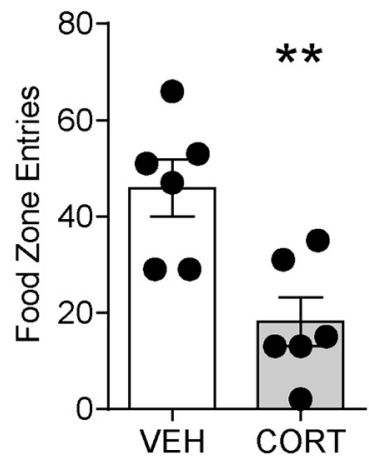

E

Open Field

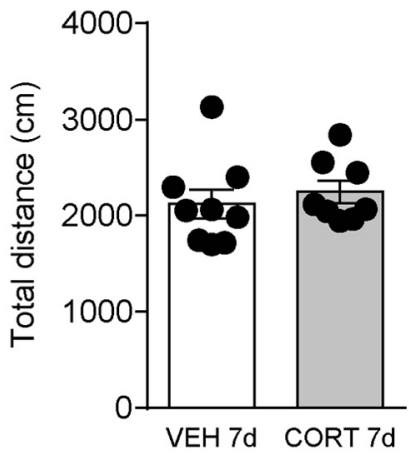

H EPM

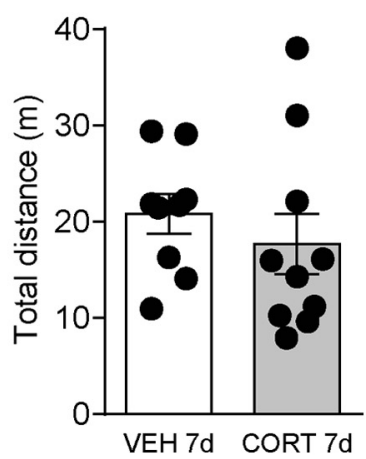

Figure 3. Seven day CORT treatment impairs reward-seeking behaviors and increases anxiety-like behavior. $\boldsymbol{A}$, Reward-seeking behavior test paradigm with HF food in the food zone. $\boldsymbol{B}$, Food zone entries, $(\boldsymbol{C})$ food zone time, and $(\boldsymbol{D})$ light box distance in vehicle or CORT mice. $\boldsymbol{E}$, Total distance in the OF test. $\boldsymbol{F}, 0$ pen arm time, $(\boldsymbol{G})$ open arm entries, and $(\boldsymbol{H})$ total distance in the EPM test. Error bars indicate SEM. Not significant, $p>0.05 . * p<0.05 . * * p<0.01$.

7 d CORT: $0.7 \pm 0.1$ per $100 \mathrm{pA}(N / n=9 / 4), t_{(17)}=2.2, p=0.038$, Fig. $5 F$ ).

A shift in excitatory and inhibitory synaptic transmission may contribute to decreased dopamine neuronal excitability. To measure the effects of EPSCs and IPSCs on the same neuron, we tested inhibitory and excitatory current reversal potentials of dopamine neurons from vehicle mice and CORT-exposed mice, respectively. The EPSC reversal potential was not significantly different between vehicle mice and CORT-exposed mice, regardless of the duration of exposure (reversal potential $\times$ CORT interaction: $F_{(1,31)}=0.38, p=0.54$; Fig. $6 A$ ). Similarly, CORT treatment did not alter the IPSC reversal potential (reversal potential $\times$ CORT interaction: $F_{(1,15)}=0.92, p=0.35$; Fig. $6 A$ ). Pooled together, the average reversal potential for EPSCs was $10 \pm 0.5 \mathrm{mV}, N / n=35 / 15$, and for IPSCs was $-68 \pm 2 \mathrm{mV}, N / n=$
19/9; Fig. 6A). Using this information, we recorded mIPSCs at $10 \mathrm{mV}$ and $\mathrm{mEPSCs}$ at $-68 \mathrm{mV}$.

The mEPSC frequency was significantly decreased in $7 \mathrm{~d}$ CORT mice (vehicle: $0.6 \pm 0.1 \mathrm{~Hz}, N / n=11 / 4$; CORT: $0.3 \pm 0.06 \mathrm{~Hz}$, $\left.N / n=11 / 7 ; t_{(20)}=2.3, p=0.034\right)$, with no significant change in mEPSC amplitude (vehicle: $14.8 \pm 0.6 \mathrm{pA}$; CORT: $15.7 \pm 0.9 \mathrm{pA}$ ), suggesting decreased glutamate release probability onto the dopamine neurons in $7 \mathrm{~d}$ CORT mice (Fig. 6B). No significant change was observed in mEPSC frequency (vehicle: $0.6 \pm 0.08 \mathrm{~Hz}$, CORT: $0.7 \pm 0.1 \mathrm{~Hz}, N / n=12 / 5$ ) or amplitude (vehicle: $15.1 \pm 0.4 \mathrm{pA}$, CORT: $14.8 \pm 0.5 \mathrm{pA}, N / n=12 / 5$ ) in $1 \mathrm{~d}$ CORT mice (Fig. $6 B$ ). To further test the locus of synaptic effect, we measured the pairedpulse ratio (PPR), a measure that correlates with release probability (Branco and Staras, 2009). There was a significant increase in PPR after 7 d but not 1 d CORT (7 d CORT: vehicle: $0.7 \pm 0.04$, 
A

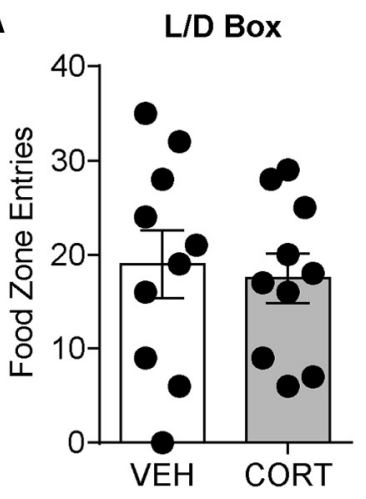

D

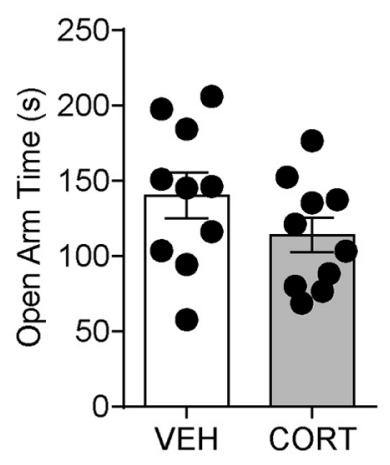

B

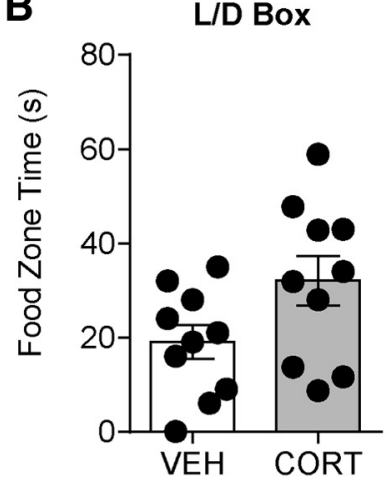

E

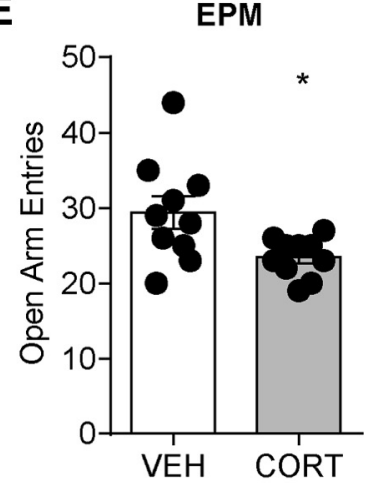

C

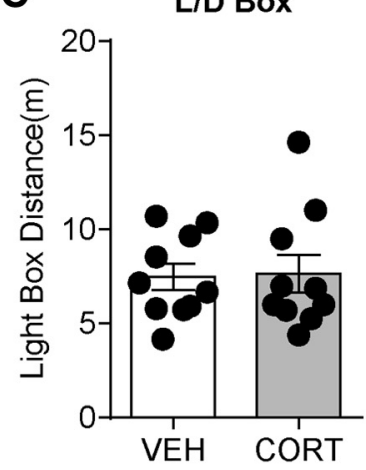

F

F EPM

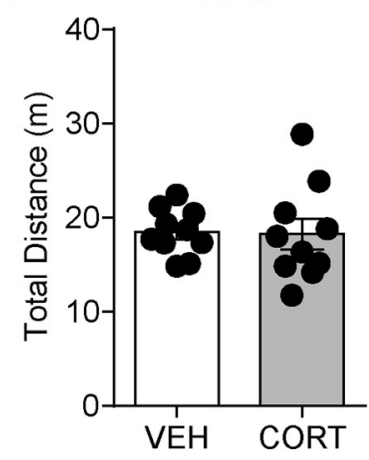

G

Open Field

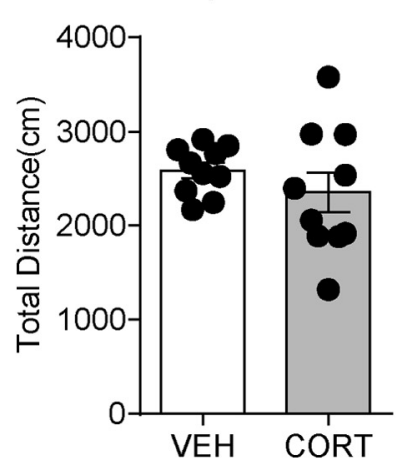

Figure 4. Reward-seeking behavior, anxiety-like behavior, and locomotor activity on 2 weeks after $7 \mathrm{~d}$ vehicle or CORT treatment. $\boldsymbol{A}$, Food zone entries. $\boldsymbol{B}$, Food zone time. $\boldsymbol{C}$, Light box distance in modified light/dark box test. $\boldsymbol{D}$, Open arm time. $\boldsymbol{E}$, Open arm entries. $\boldsymbol{F}$, Total distance in EPM. $\mathbf{G}$, Total distance in OF test. Error bars indicate SEM. $* p<0.05$.

$N / n=13 / 6 ;$ CORT: $0.8 \pm 0.02, N / n=13 / 8 ; t_{(24)}=2.4, p=0.024 ; 1 \mathrm{~d}$ CORT: vehicle: $0.7 \pm 0.03, N / n=10 / 7$; CORT: $0.7 \pm 0.02, N / n=$ $7 / 5$ ), suggesting a decrease in presynaptic release probability in $7 \mathrm{~d}$ CORT mice (Fig. 6D).

Similarly, mIPSC frequency was decreased in $7 \mathrm{~d}$ CORT mice (vehicle: $2.3 \pm 0.3 \mathrm{~Hz}$; CORT: $1.3 \pm 0.2 \mathrm{~Hz} ; t_{(20)}=2.6, p=0.017$ ) with no significant change in the amplitude (vehicle: $17.8 \pm 0.6$ pA; CORT: $18.2 \pm 0.5 \mathrm{pA}$ ) (Fig. 6C). Because both excitatory and inhibitory synaptic transmission was decreased in $7 \mathrm{~d}$ CORT mice, we did not see a significant change in excitatory/inhibitory (E/I) ratio (vehicle: $0.3 \pm 0.04, N / n=11 / 4$; CORT: $0.2 \pm 0.04$, $N / n=11 / 7 ; p=0.25$ ). In $1 \mathrm{~d}$ CORT mice, mIPSC frequency (vehicle: $1.9 \pm 0.3 \mathrm{~Hz}$, CORT: $1.7 \pm 0.2 \mathrm{~Hz}, n=12 / 5$ ), amplitude (vehicle: $18.2 \pm 0.9 \mathrm{pA}$, CORT: $17.9 \pm 0.5 \mathrm{pA}, N / n=12 / 5$ ) (Fig. $6 C$ ), or E/I ratio (vehicle: $0.5 \pm 0.1$, CORT: $0.5 \pm 0.1, N / n=12 / 5$ ) was not significantly different.
We measured the relative contribution of AMPAR and NMDAR EPSCs after vehicle or CORT treatment. The AMPAR/ NMDAR ratio was not significant different between vehicle and CORT mice regardless of exposure duration (7 d CORT, vehicle: $0.6 \pm 0.04, N / n=13 / 4$, CORT: $0.6 \pm 0.1, N / n=9 / 5, t_{(20)}=0.15$, $p=0.88$; $1 \mathrm{~d}$ CORT, vehicle: $0.6 \pm 0.05, n=8 / 4$, CORT: $0.5 \pm$ $0.04, n=12 / 5, t_{(18)}=1.8, p=0.08$; Fig. $7 A$ ), suggesting there was no relative change in number or function of postsynaptic excitatory receptors.

Decreased presynaptic release probability may result from an increased endocannabinoid-mediated retrograde inhibition (Busquets-Garcia et al., 2018). In response to glucocorticoids, endocannabinoid induces a rapid suppression of synaptic transmission in the hypothalamus and the basolateral amygdala (Di et al., 2003, 2016). To test whether CORT treatment alters endocannabinoid tone, evoked 
A

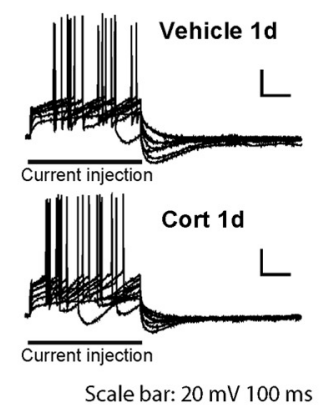

B

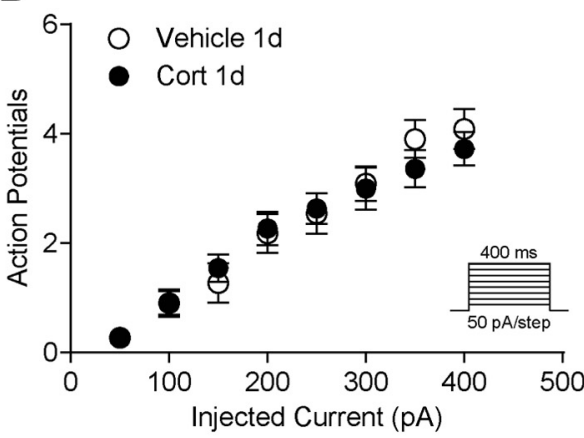

C

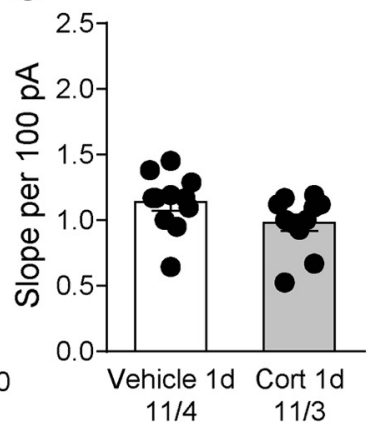

D

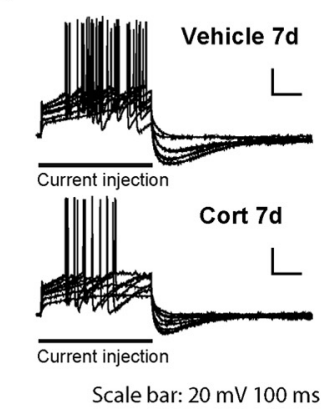

E

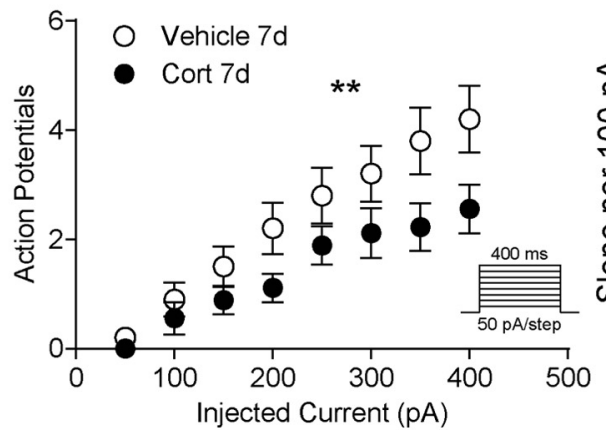

$\mathbf{F}$

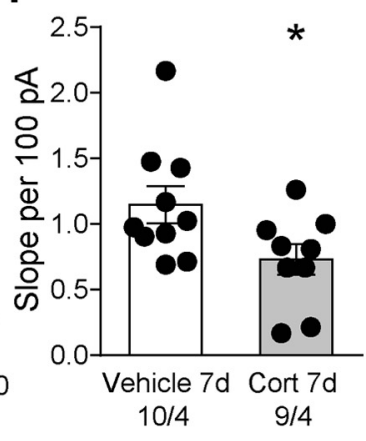

Figure 5. Decreased VTA dopamine neuron excitability in $7 \mathrm{~d}$ CORT mice. $\boldsymbol{A}$, Representative traces for neuron spikes elicited by current steps after $1 \mathrm{~d}$ treatment of vehicle or CORT. $\boldsymbol{B}$, The number of action potentials in response to current injection in $1 \mathrm{~d}$ treatment mice. $\boldsymbol{C}$, The slope of the number of spikes per $100 \mathrm{pA}$. $\boldsymbol{D}$, Representative traces for neuron spikes elicited by current steps after $7 \mathrm{~d}$ treatment of vehicle or CORT. $\boldsymbol{E}$, The number of action potentials in response to current injection in $7 \mathrm{~d}$ treatment mice. $\boldsymbol{F}$, The slope of the number of spikes per 100 pA in $7 \mathrm{~d}$ treatment mice. $* p<0.05 . * * p<0.01$.

EPSCs were measured in the presence of a neutral CB1 receptor antagonist, NESS-0327. Application of NESS-0327 $(0.5 \mu \mathrm{M})$ did not significantly change EPSC amplitude onto VTA neurons of vehicle or $1 \mathrm{~d}$ CORT mice (vehicle+NESS0327: $93 \pm 2 \%$ of baseline, $N / n=6 / 3$; CORT + NESS-0327: $101 \pm 4 \%$ of baseline, $N / n=8 / 6, t_{(12)}=1.8, p=0.10$; Fig. $7 B$ ) or of vehicle or $7 \mathrm{~d}$ CORT mice (vehicle + NESS-0327: $99 \pm 3 \%$ of baseline, $N / n=7 / 6$; CORT +NESS-0327: $99 \pm 5 \%$ of baseline, $N / n=8 / 7, t_{(12)}=0.072, p=0.94$; Fig. $\left.7 C\right)$. Thus, decreased presynaptic release probability was not because of increased endocannabinoid tone.

Chronic CORT treatment increased somatodendritic dopamine, and D2R antagonist sulpiride restores mEPSCs frequency and neuronal excitability

Somatodendritic dopamine regulates dopamine neuronal excitability through dopamine D2R-mediated autoinhibition (Jeziorski and White, 1989) and neurotransmission at presynaptic terminals expressing D2Rs (Pickel et al., 2002). To test whether 7 d CORT altered somatodendritic dopamine concentration $[\mathrm{DA}]_{\mathrm{o}}$, we applied electrical stimulation $(40 \mathrm{~Hz}, 20$ pulses) to evoke dopamine release in VTA slices and measured the relative extracellular dopamine concentration $\left([\mathrm{DA}]_{\mathrm{o}}\right)$ using fast-scan cyclic voltammetry (Fig. $8 A, B)$. Evoked $[\mathrm{DA}]_{\mathrm{o}}$ was significantly greater in $7 \mathrm{~d}$ CORT-treated compared with vehicle-treated mice (vehicle: $55 \pm 6 \mathrm{nM}, N / n=29 / 5$; CORT: $82 \pm 9 \mathrm{~nm}, N / n=18 / 3$, Mann-Whitney test, $p=0.003$; Fig. $8 B, C$ ), indicating that chronic CORT treatment increases somatodendritic dopamine.

We next examined whether decreased neuronal transmission and excitability resulted from enhanced D2R signaling by elevated somatodendritic dopamine. Bath application of the D2R antagonist sulpiride $(5 \mu \mathrm{M})$ restored mEPSC interevent interval cumulative probability (Fig. $8 E$; Kolmogorov-Smirnov test) and mEPSCs frequency ((Fig. $8 F)$ in VTA neurons from CORT mice (main effect of CORT: $F_{(1,20)}=20.15, p=0.0002$ and sulpiride: $F_{(1,20)}=14.14, p=0.0012$; vehicle + aCSF: $0.9 \pm 0.07 \mathrm{~Hz}, N / n=$ 8/5; CORT +aCSF: $0.4 \pm 0.04 \mathrm{~Hz}, N / n=5 / 4$; vehicle + sulpiride: $1.2 \pm 0.2 \mathrm{~Hz}, \mathrm{~N} / n=6 / 6$; CORT + sulpiride: $0.9 \pm 0.1 \mathrm{~Hz}, \mathrm{~N} / n=5 / 3$; Fig. $8 D-F)$. There was no significant interaction between CORT and sulpiride treatment on mEPSC amplitude (vehicle+aCSF: $18 \pm 1 \mathrm{pA}, N / n=8 / 5$; CORT $+\mathrm{aCSF}: 17 \pm 0.9 \mathrm{pA}, N / n=5 / 4$; vehicle + sulpiride: $15 \pm 0.3 \mathrm{pA}, N / n=6 / 6$; CORT + sulpiride: $16 \pm 0.7$ $\left.\mathrm{pA}, N / n=5 / 3 ; F_{(1,20)}=0.68, p=0.42\right)($ Fig. $8 G)$. Together, these data indicate that D2Rs are at least partially required for decreased release probability of glutamate on to VTA dopamine neurons of $7 \mathrm{~d}$ CORT-treated mice.

To test the impact of D2R signaling blockade on neuronal excitability, we measured the spike number elicited by depolarizing current steps with or without application of sulpiride. Sulpiride did not change firing $\left(t_{(8)}=0.4, p=0.68\right)$ or slope of the frequency-current plot, suggesting that, in vehicle-treated mice, there is no dopaminergic tone acting at $\mathrm{D} 2 \mathrm{R}$ receptors (Fig. $8 \mathrm{H}$ ). In contrast, sulpiride significantly increased the excitability $(\mathrm{CORT} \times$ sulpiride interaction: repeated-measures ANOVA: $\left.F_{(7,56)}=4.8, p=0.0003\right)$ and frequency-current slope of VTA neurons from $7 \mathrm{~d}$ CORT mice (slope: $1.4 \pm 0.2$ per $100 \mathrm{pA}, N / n=9 / 4-1.6 \pm 0.1$ per $100 \mathrm{pA}, N / n=9 / 4$, $\left.t_{(8)}=2.6, p=0.031\right)$ (Fig. $8 I$ ).

D2R signaling blockade in the VTA restores food-seeking behaviors and alleviates anxiety-like behavior, but does not rescue motivated behavior

To investigate the effect of a D2R antagonist on food-seeking behaviors, we infused with either aCSF or sulpiride intra-VTA 
A
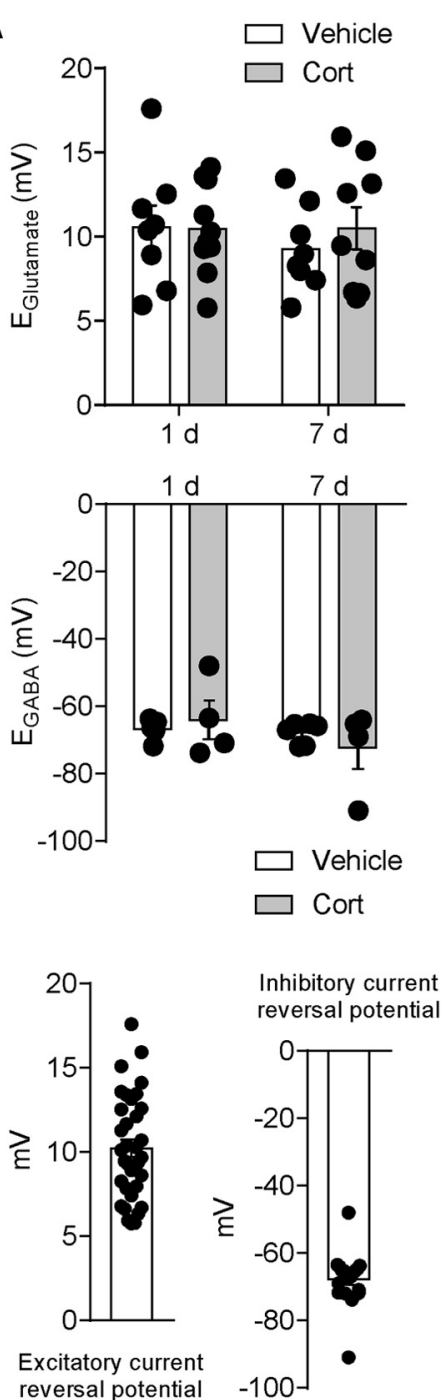

D

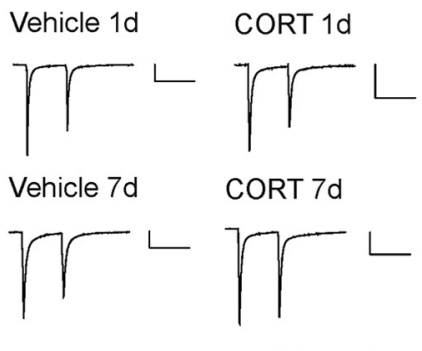

B

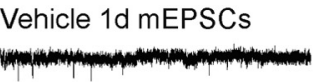

Cort $1 \mathrm{~d}$ mEPSCs
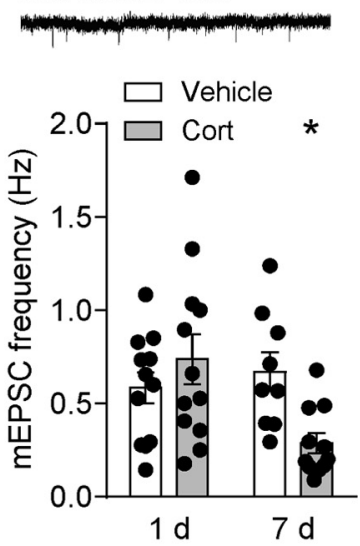

C
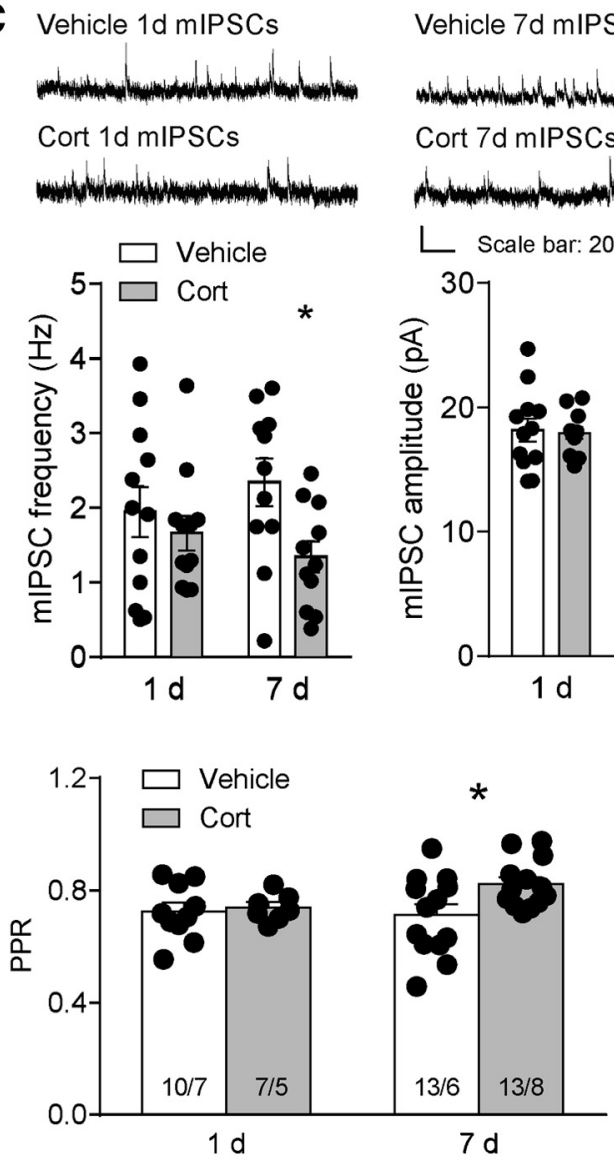

Vehicle 7d mEPSCs

Cort 7d mEPSCs

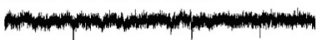

L Scale bar: 20 pA; $256 \mathrm{~ms}$

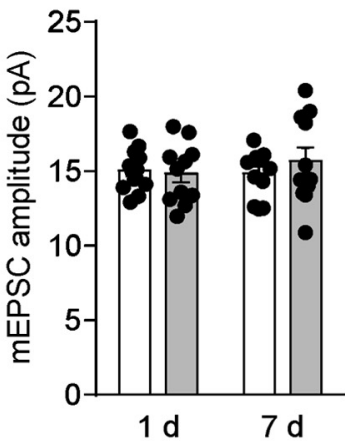

Vehicle 7d mIPSCs

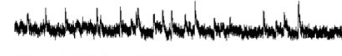

Cort $7 \mathrm{~d}$ mIPSCs

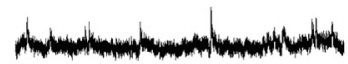

L_ Scale bar: 20 pA; $256 \mathrm{~ms}$

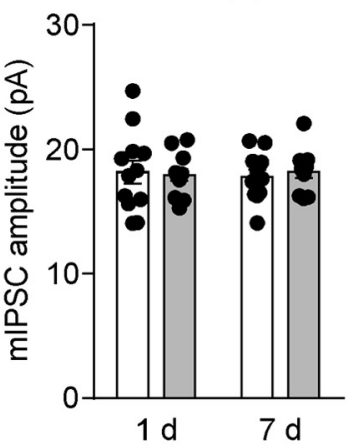

Scale bar: $100 \mathrm{pA} ; 50 \mathrm{~ms}$

Figure 6. Decreased VTA dopamine neuron presynaptic glutamate release in $7 \mathrm{~d}$ CORT mice. $A$, Excitatory reversal potential (top) and inhibitory reversal potential (middle) in vehicle and CORT mice on 1 and $7 \mathrm{~d}$ treatment, respectively. Data were pooled together to calculate excitatory current reversal potential and inhibitory current reversal potential (bottom). $\boldsymbol{B}$, Representative traces, frequency, and amplitude for mEPSCs in vehicle and CORT mice. C, Representative traces, mIPSC frequency, and mIPSC amplitude for mIPSCs in vehicle and CORT mice. $\mathbf{D}$, Left, Representative traces for PPR. Right, PPR in $1 \mathrm{~d}$ and $7 \mathrm{~d}$ treatment mice. Error bars indicate SEM. $* p<0.05$.

before the modified light-dark box test (Fig. 9A). Decreased food zone entries in $7 \mathrm{~d}$ CORT mice (vehicle+aCSF: $30 \pm 5, n=13$; CORT+aCSF: $13 \pm 3, n=12$ ) were restored by intra-VTA sulpiride with a main effect of CORT treatment $\left(F_{(1,41)}=5.69\right.$, $p=0.016)$ and sulpiride infusion $\left(F_{(1,41)}=6.28, p=0.022\right.$; Fig. $9 B)$. There was no interaction between CORT treatment and drug treatment on food zone time $\left(F_{(1,41)}=0.04, p=0.85\right.$, vehicle + aCSF: $27 \pm 5 \mathrm{~s}, n=13$; CORT + aCSF: $10 \pm 3$ s, $n=12$; vehicle+sulpiride: $40 \pm 10 \mathrm{~s}, n=11$; CORT + sulpiride: $25 \pm 6 \mathrm{~s}$, $n=9$; Fig. $9 C)$, with main effects of $\operatorname{CORT}\left(F_{(1,41)}=6.11\right.$, $p=0.02)$ and intra-VTA sulpiride $\left(F_{(1,41)}=4.8, p=0.03\right)$. Vehicle and $7 \mathrm{~d}$ CORT mice treated with either aCSF or sulpiride had no significant difference in distance traveled in the light box (vehicle+aCSF: $12 \pm 1 \mathrm{~m}, n=13$; CORT + aCSF: $9 \pm 0.9 \mathrm{~m}$, $n=12$; vehicle + sulpiride: $15 \pm 2 \mathrm{~m}, n=11$; CORT + sulpiride: $16 \pm 3 \mathrm{~m}, n=9$, main effect of CORT: $F_{(1,41)}=0.35, p=0.6$; Fig. $9 D)$. In addition, sulpiride did not change total distance traveled in the OF test (vehicle+aCSF: $39 \pm 4 \mathrm{~m}, n=8$; CORT+aCSF: 
A
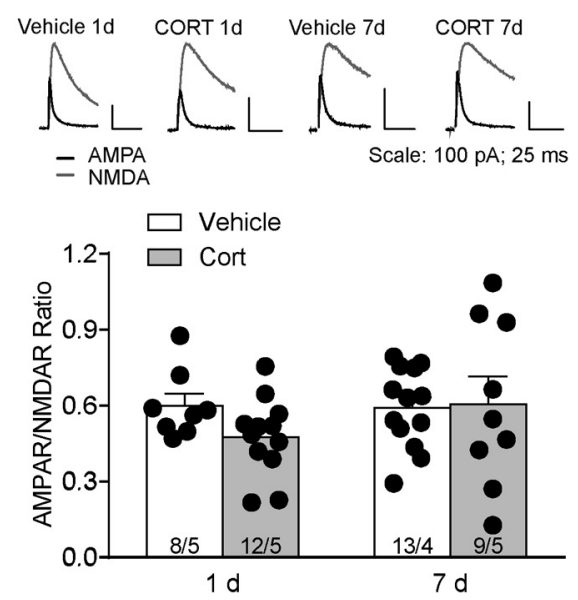

B

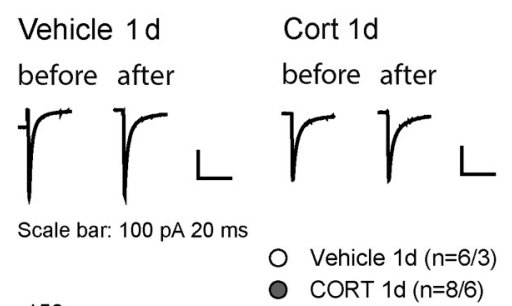

C

Vehicle 7d Cort 7d

before after before after

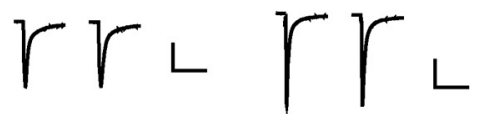

Scale bar: $100 \mathrm{pA} 20 \mathrm{~ms}$

Vehicle $7 d(n=7 / 6)$

CORT $7 d(n=8 / 7)$

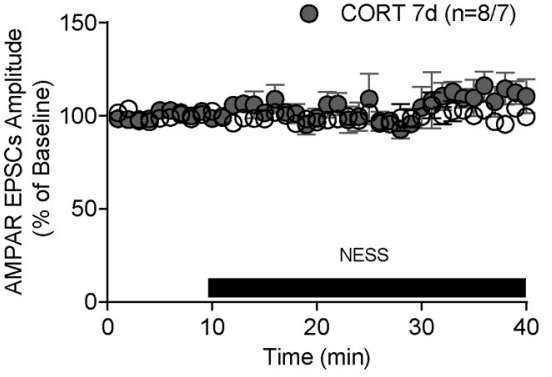

Figure 7. No change in AMPAR/NMDAR ratio and endocannabinoid tone in CORT mice. $A$, Top, Representative traces for AMPAR/NMDAR ratio. Bottom, AMPAR/NMDAR ratio in vehicle and CORT mice. $\boldsymbol{B}$, Top, Representative traces for evoked EPSCs before and after the application of CB1R antagonist NESS in $1 \mathrm{~d}$ vehicle or CORT mice. Bottom, Evoked EPSC amplitude in the presence of CB1R antagonist. C, Top, Representative traces for evoked EPSCs before and after the application of CB1R antagonist NESS in 7 d vehicle or CORT mice. Bottom, Evoked EPSC amplitude in the presence of CB1R antagonist. Error bars indicate SEM.

$39 \pm 3 \mathrm{~m}, n=8$; vehicle + sulpiride: $41 \pm 3 \mathrm{~m}, n=9$; CORT + sulpiride: $42 \pm 4 \mathrm{~m}, n=8, F_{(1,29)}=0.032, p=0.86$, main effect of CORT: $F_{(1,29)}=0.012, p=0.9$; Fig. $\left.9 E, F\right)$. These data indicate that $\mathrm{D} 2 \mathrm{R}$ inhibition can restore food-seeking with no change in locomotor activity in $7 \mathrm{~d}$ CORT mice.

To further test whether the restoration of food-seeking behaviors is because of alleviated anxiety-like behavior or normalized motivated behavior, we tested vehicle and CORT mice in the EPM test (Fig. 10A) and operant chamber (Fig. 10E) with intraVTA aCSF or sulpiride infusion, respectively. Sulpiride restored CORT-induced reductions in open arm time (vehicle + aCSF: $115 \pm 11 \mathrm{~s}, n=8$; CORT + aCSF: $60 \pm 13 \mathrm{~s}, n=8$; vehicle + sulpiride: $102 \pm 10 \mathrm{~s}, n=9$; CORT + sulpiride: $114 \pm 18 \mathrm{~s}, n=8, F_{(1,29)}=$ 6.28, $p=0.018$; Fig. $10 B$ ) and open arm entries (vehicle + aCSF: $37 \pm 3, n=8$; CORT + aCSF: $23 \pm 3, n=8$; vehicle+sulpiride: $31 \pm 3, n=9$; CORT+ sulpiride: $40 \pm 5, n=8, F_{(1,29)}=10.88$, $p=0.0026$; Fig. $10 C$ ), with no effects on total distance (vehicle+ aCSF: $19 \pm 2 \mathrm{~m}, n=8$; CORT + aCSF: $19 \pm 1 \mathrm{~m}, n=8$; vehicle + sulpiride: $22 \pm 1 \mathrm{~m}, n=9$; CORT + sulpiride: $22 \pm 2 \mathrm{~m}, n=8$, $F_{(1,29)}=0.015, p=0.90$; Fig. $\left.10 D\right)$ in the EPM test. PR schedule was used in operant chamber test to assess motivated behavior. Breakpoint was significantly less in CORT mice (vehicle + aCSF: $42 \pm 3, n=7$; CORT + aCSF: $37 \pm 6, n=8$; vehicle+sulpiride: $49 \pm 5, n=9$; CORT + sulpiride: $35 \pm 2, n=8$, CORT main effect, $F_{(1,28)}=4.45, p=0.044$; Fig. $\left.10 F\right)$. However, sulpiride had no main effect on instrumental performance $\left(F_{(1,28)}=0.30, p=0.59\right)$, and no interaction between CORT and sulpiride was observed $\left(F_{(1,28)}=1.20, p=0.28\right)$. These results suggest that $\mathrm{D} 2 \mathrm{R}$ signaling blockade in the VTA of CORT mice can alleviate anxiety-like behavior but does not rescue motivated behavior.

\section{Discussion}

Here, we report chronic CORT treatment impairs food-seeking behavior and elevates anxiety-like behavior in mice. Furthermore, we observed decreased excitability and synaptic transmission onto dopamine neurons of chronic CORT-treated mice. This was likely mediated by increased somatodendritic dopamine-activating presynaptic and postsynaptic D2Rs (schematic, Fig. 11). Blockade of D2Rs restored food-seeking behavior in a mildly aversive environment and alleviated anxiety-like behavior in chronic CORT-exposed mice. Together, CORT-induced neuronal disruptions in a key region mediating reward-seeking and may underlie CORT-induced anhedonia and neuropsychiatric sequelae.

CORT induced altered reward-seeking behavior, elevated anxiety-like behavior and metabolic dysfunction in mice While sated vehicle-treated mice will explore and engage with palatable food in the light box, consistent with previous work establishing this model of nonhomeostatic feeding (Teegarden and Bale, 2007; Cottone et al., 2012; Liu et al., 2016), mice treated with $7 \mathrm{~d}$ CORT have reduced food-seeking in this paradigm. It is likely that enhanced anxiety-like behavior contributed to reduced food approach behavior, given that, in this food-seeking model, mice must overcome their innate aversion to light open spaces. Alternatively, this may be because of increased satiety in CORTtreated mice as they consume more home cage food. While others have demonstrated that prolonged CORT treatment (4 weeks) reduces home cage locomotor activity (Karatsoreos et al., 2010), 7 d CORT-treated mice do not exhibit locomotor impairment. Regardless, these results suggest that $7 \mathrm{~d}$ CORT treatment induces an altered motivational state to reduce foodseeking. Indeed, exogenous CORT administration in humans reveals a downregulation of the limbic reward system, and thereby diminishes reward anticipation and motivational processing (Tidey and Miczek, 1996; Berton et al., 2006).

Cushing's syndrome is associated with elevated CORT, which presents in males at a younger age with more significant CORT elevation and more severe clinical symptoms (Kleen et al., 2006). Chronic oral CORT treatment in mice has been characterized as a model of Cushing's syndrome (Kinlein et al., 2017). Prolonged CORT treatment initiated at postnatal day 35 leads to an initial drop in weight during the first week of treatment followed by significant weight gain (Karatsoreos et al., 2010). This is associated with increased adiposity as well as impaired glucose tolerance (Karatsoreos et al., 2010; Kinlein et al., 2017). Consistent with these studies, our results show that $7 \mathrm{~d}$ CORT treatment in periadolescent male mice have decreased weight gain, lower basal glucose level, hyperphagia, and increased water intake. 
A

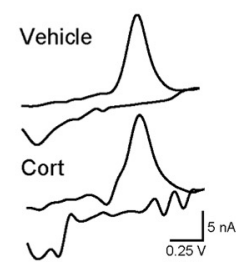

D

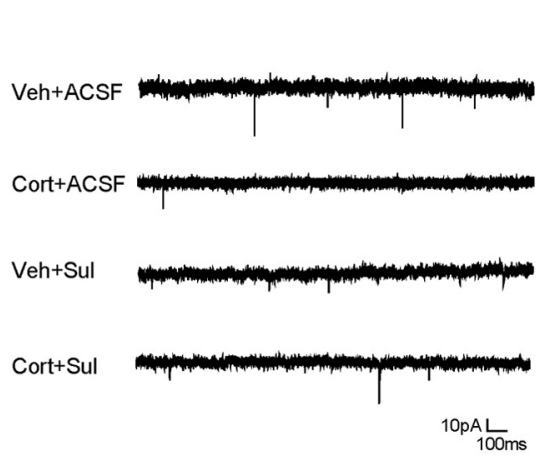

F
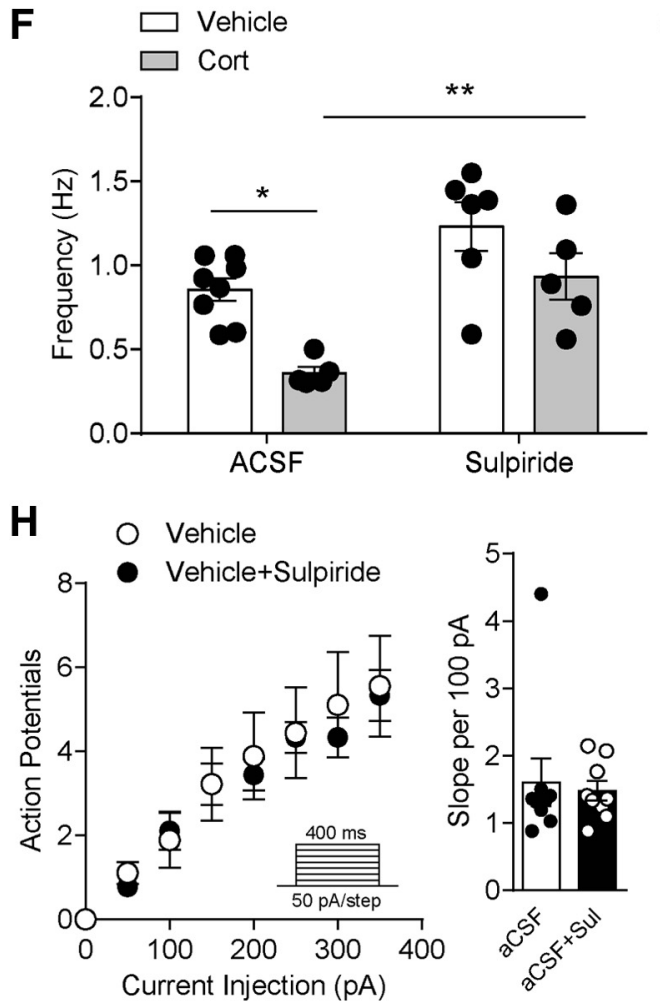

B

E

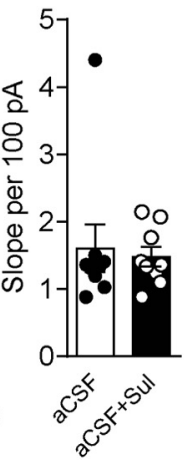

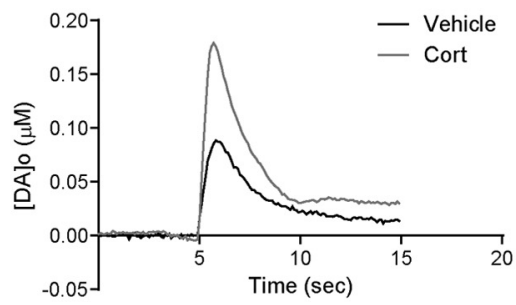

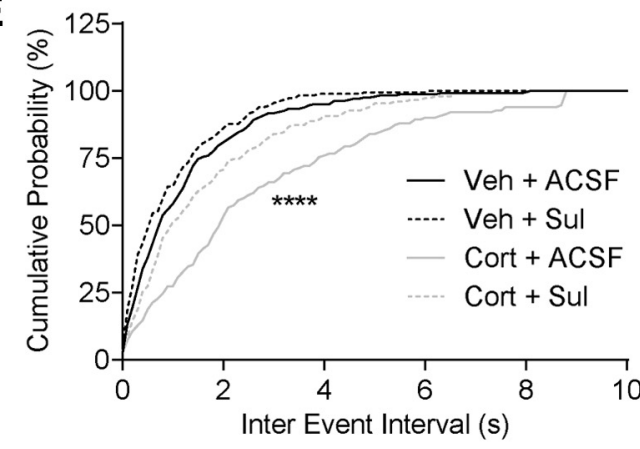

G

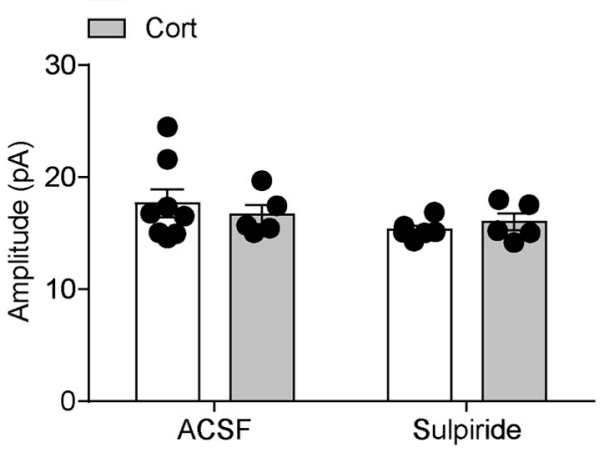

I

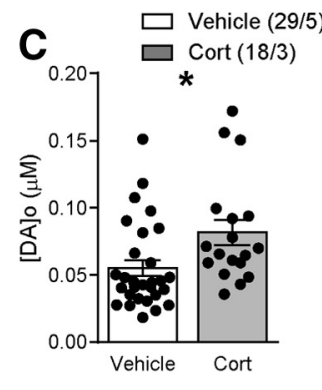


A

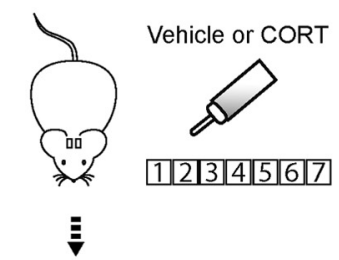

Intra VTA aCSF or Sulpiride<smiles>CC=CC1CCCCC1CCCC</smiles>

B

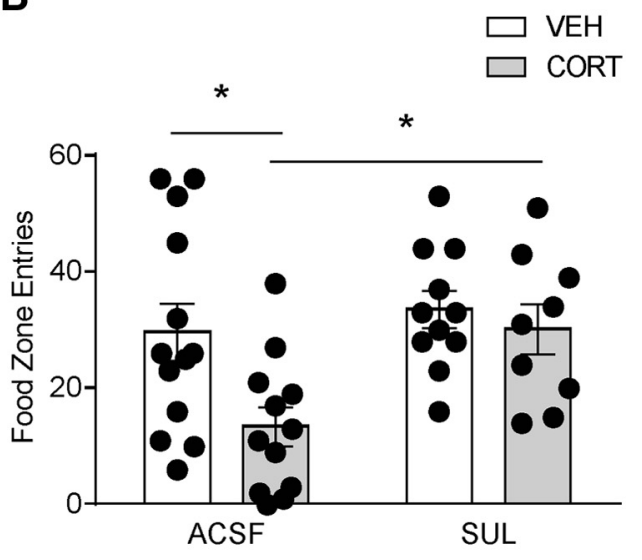

C

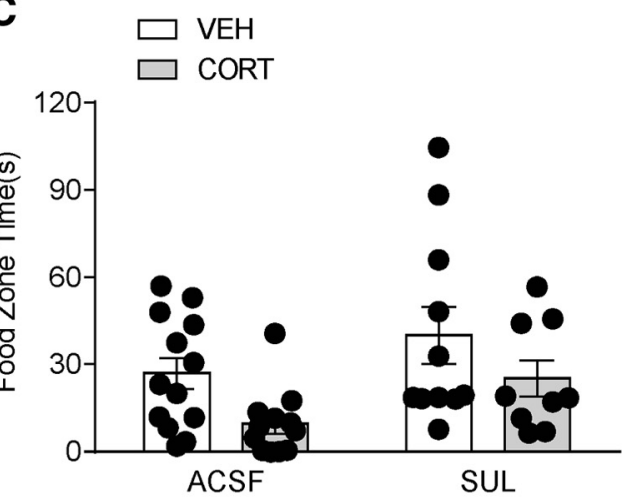

E

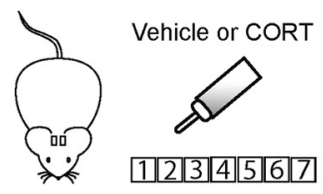

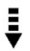

Intra VTA aCSF or Sulpiride

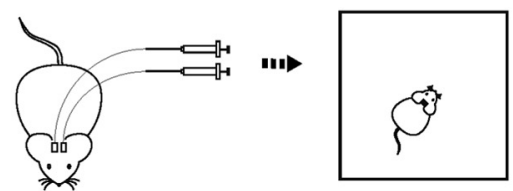

D

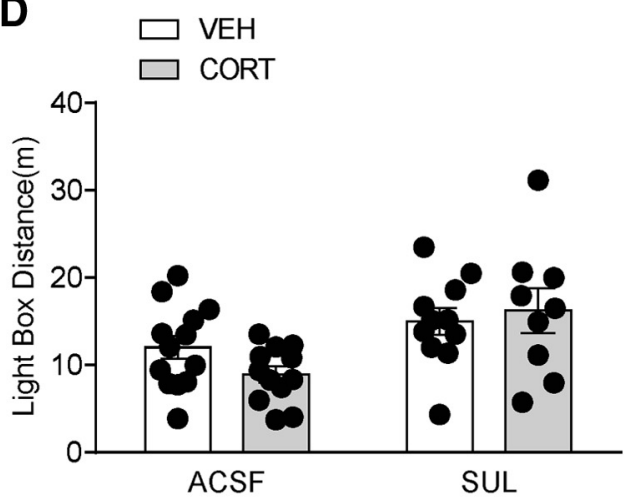

F

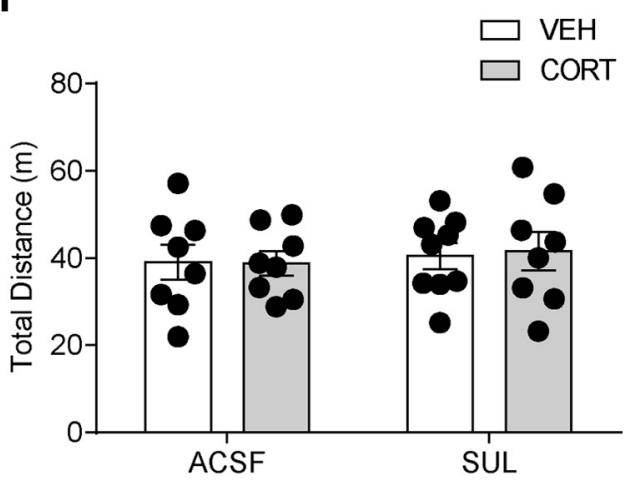

Figure 9. Decreased reward-seeking behavior can be reversed by intra-VTA sulpiride infusion with no change in locomotor activity. $\boldsymbol{A}$, Flowchart for reward-seeking behavior test, (B) food zone entries, $(\boldsymbol{C}$ ) food zone time, and (D) distance traveled in the light box in vehicle and CORT mice with either intra-VTA aCSF or sulpiride infusion. $\boldsymbol{E}$, Flowchart for OF test. $\boldsymbol{F}$, Total distance traveled in the open field with either intra-VTA aCSF or sulpiride infusion. Error bars indicate SEM. $* p<0.05$.

treatment markedly decreased excitability of VTA dopamine neurons. CORT may change neuronal excitability by directly targeting GR- or MR-expressing dopamine neurons to influence cellular activity or indirectly acting on upstream brain regions targeting VTA neurons. After chronic CORT treatment, hypothalamic GR-expressing CRH neurons exhibit reduced excitability (Bittar et al., 2019), whereas hippocampal neurons have enhanced excitatory postsynaptic receptor responses (Karst and Joels, 2005). Activation of these projections could drive VTA dopamine release. Because chronic CORT treatment increased somatodendritic dopamine, it is possible that enhanced dopamine targets D2R to suppress VTA dopamine firing rate (Jeziorski and White, 1989). Indeed, inhibition of D2R with sulpiride in CORT-treated mice restored the excitability of dopamine neurons. Notably, D2Rs are expressed on extrasynaptic dendrites of VTA dopamine neurons near excitatory synapses (Pickel et al., 2002); therefore, D2R activation via somatodendritic dopamine is likely to dampen the influence of excitatory inputs.

Dopamine neurons in the VTA innervate brain regions that are critical for emotional processing (Russo and Nestler, 2013) and mediate symptoms of anxiety, including NAc, amygdala, 
A
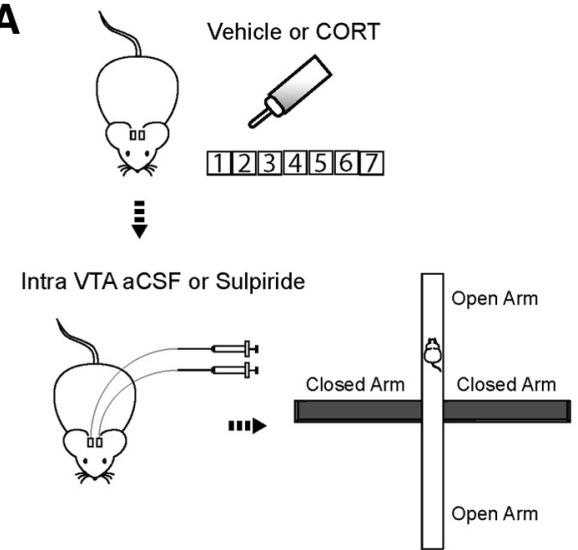

C $\square$ VEH

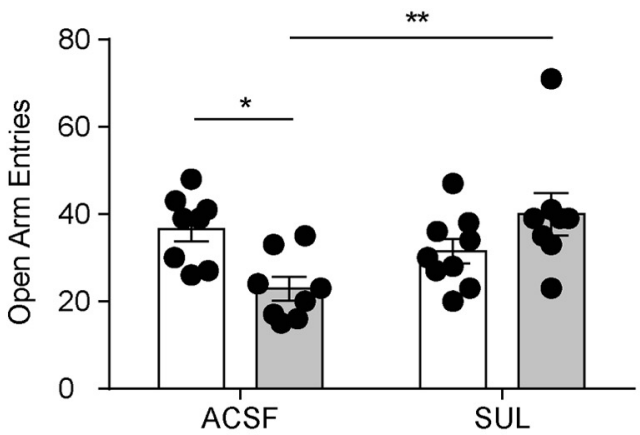

E

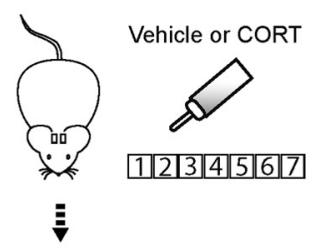

Intra VTA aCSF or Sulpiride

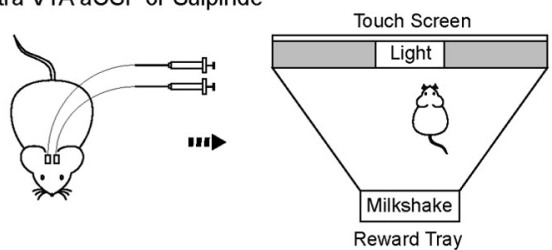

B
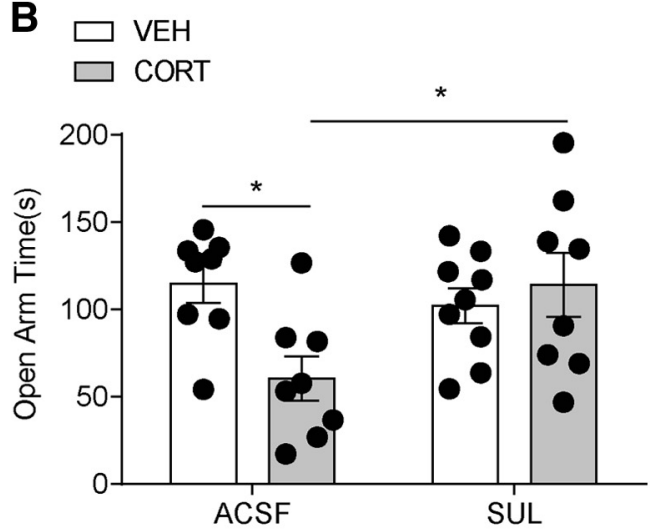

D
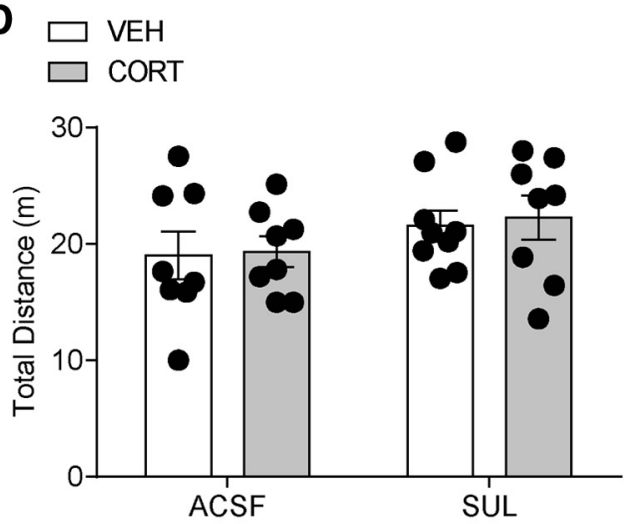

$F$

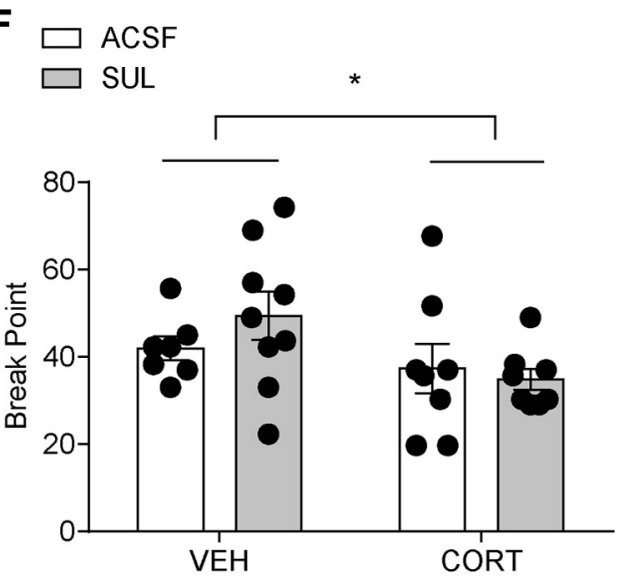

Figure 10. Intra-VTA sulpiride infusion alleviates anxiety-like behavior but does not rescue motivated behavior. $\boldsymbol{A}$, Flowchart for EPM test, $(\boldsymbol{B})$ open arm time, $(\boldsymbol{C})$ open arm entries, and $(\boldsymbol{D})$ total distance for vehicle and CORT mice with either intra-VTA aCSF or sulpiride infusion. $\boldsymbol{E}$, Flowchart for PR test. $\boldsymbol{F}$, Breakpoint for vehicle and CORT mice with either intra-VTA aCSF or sulpiride infusion. Error bars indicate SEM. $* p<0.05 . * * p<0.01$.

PFC, and hippocampus. Anxiety-like responses, such as reduced open arm time in the EPM test and decreased head-dipping in the hole-board test, can be modulated by dopaminergic receptors in the amygdala (Bananej et al., 2012), hippocampus (Zarrindast et al., 2010; Nasehi et al., 2011), and PFC (Broersen et al., 2000; Wall et al., 2004). Impaired dopamine neuronal activation can result in enhanced anxiety-like behaviors (Zweifel et al., 2011). Therefore, impaired dopamine neuron excitability in CORT mice can contribute to an elevated anxiety-like phenotype via a variety of corticolimbic circuits.

Systemic dexamethasone or forced swim test increases the AMPAR/NMDAR ratio of VTA dopamine neurons, an effect blocked by the GR/progesterone receptor inhibitor, RU486 (Saal et al., 2003). However, while $1 \mathrm{~d}$ CORT self-administration was not sufficient to change synaptic transmission, chronic CORT treatment reduced both excitatory and inhibitory synaptic transmission. Because there was no shift in the E/I balance onto dopamine neurons of CORT-treated mice, the decrease in firing rate is unlikely because of an altered synaptic input, but rather an intrinsic change to dopamine neurons. Decreased mEPSC frequency, but not amplitude, and a paired-pulse facilitation of glutamate release onto dopamine neurons was consistent with a change in presynaptic release probability. However, this effect was not because of elevated endocannabinoid tone. By contrast, $\mathrm{D} 2 \mathrm{R}$ inhibition reversed the $7 \mathrm{~d}$ CORT-induced suppression of mEPSC frequency, suggesting that CORT-induced elevated 


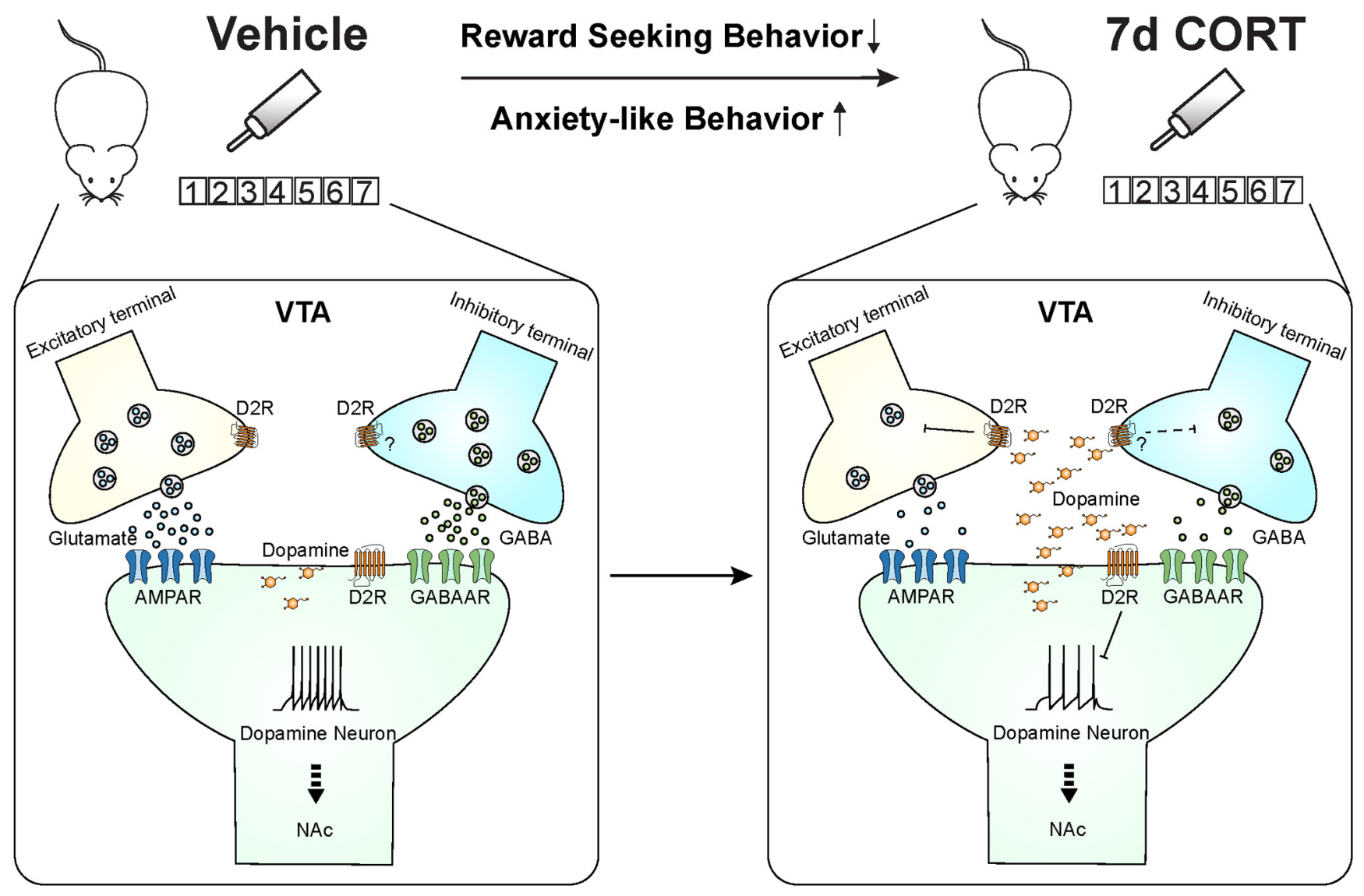

Figure 11. Schematic diagram showing mechanism underlying $7 \mathrm{~d}$ CORT treatment impairment of food-seeking behavior and anxiety-like behavior. A critical node implicated in this dysfunction is the VTA. Decreased excitability and synaptic transmission onto the dopamine neurons in CORT mice are likely mediated by increased somatodendritic dopamine activating presynaptic and postsynaptic D2R signaling.

somatodendritic dopamine could act at D2Rs to suppress release probability. The presynaptic inhibition could be general or specific to some afferents depending on the expression of D2R. D2Rs are primarily expressed on dopamine neuronal dendrites and somata but have also been localized to nondopaminergic axon fibers in the VTA as well as astrocytes (Sesack et al., 1994). The restoration of synaptic transmission by D2R inhibition rules out possible synaptic scaling from CORT exposure as has been observed in hypothalamic CRH neurons (Bittar et al., 2019). Together, chronic CORT increases somatodendritic dopamine, which acts at D2R to suppress excitatory synaptic transmission onto dopamine neurons. Although there is no direct evidence for D2R expression on inhibitory terminals in the VTA, one study suggests that activation of $\mathrm{D} 2 \mathrm{R}$ at inhibitory terminals facilitates endocannabinoid-mediated long-term depression in the VTA DA neurons (Pan et al., 2008). Further study should test the mechanisms of how CORT treatment suppresses inhibitory synapses and determine the D2R-sensitive excitatory inputs suppressed with chronic CORT exposure.

Inhibition of D2Rs in the VTA also restored the CORTinduced suppression of food-seeking, consistent with previous studies showing VTA D2R availability is inversely associated with incentive motivation (de Jong et al., 2015) and novelty seeking (Zald et al., 2008; Tournier et al., 2013). Previous reports have indicated that systemic administration of a high $(50 \mathrm{mg} / \mathrm{kg})$ but not low $(25 \mathrm{mg} / \mathrm{kg})$ dose of sulpiride decreases rats' responding under a PR schedule of reinforcement (Heath et al., 2015; Sakamoto et al., 2015). Notably, we did not observe a significant effect of intra-VTA sulpiride infusion on breakpoint in control mice. Consistent with this, mice lacking D2Rs show similar acquisition in self-administration for natural reward compared with WT mice (Holroyd et al., 2015). Because VTA D2R blockade alleviated anxiety-like behavior but did not rescue instrumental performance, the antianxiety effect of D2R blockade likely contributes to restored reward-seeking behavior. Further study needs to tackle which pathway is involved in the CORT-induced suppression of motivated behavior.

CORT is a mediator of stress responses and provides feedback to the CNS that controls cognitive and emotional processing. Although CORT administration through the drinking water does not fully represent a physiological stress response, it can explicitly manipulate CORT concentration and recapitulate chronic stress in critical aspects of the neuroendocrine response, justifying it a valuable approach for understanding the stressrelated pathology. Acute stress modulates dopamine neurons in a projection-specific way. Although the effects are complex, one prominent observation is increased dopamine release in the NAc and PFC in response to an acute stressor (Tidey and Miczek, 1996; Young, 2004; Lammel et al., 2011). Chronic stress induced mood and motivation deficits can be attributed to dysfunction of mesolimbic dopamine pathways (Berton et al., 2006; Mehta et al., 2010). Consistent with our study showing decreased DA neuronal excitability in chronic CORT mice, long-term psychological stress in humans dampens striatal dopaminergic function (Bloomfield et al., 2019). Therefore, D2 autoinhibition of DA release can be tested as a potential underlying mechanism in chronic stress-induced deficits. 
In conclusion, chronic CORT decreases neuronal excitability in the VTA and food-seeking behavior in a mildly aversive environment. This effect requires activation of D2Rs likely through elevated somatodendritic dopamine. Although food approach behaviors are largely restored by blocking D2R signaling in the current paradigm, the full recovery of reward-seeking behavior may also require involvement of other brain regions that may have distinct mechanisms influenced by chronic CORT. These results suggest that neuropsychiatric side effects of CORT medication related to reward dysfunction may be ameliorated with D2R antagonists.

\section{References}

Bananej M, Karimi-Sori A, Zarrindast MR, Ahmadi S (2012) D1 and D2 dopaminergic systems in the rat basolateral amygdala are involved in anxiogenic-like effects induced by histamine. J Psychopharmacol 26:564-574.

Berry JN, Saunders MA, Sharrett-Field LJ, Reynolds AR, Bardo MT, Pauly JR, Prendergast MA (2016) Corticosterone enhances N-methyl-D-aspartate receptor signaling to promote isolated ventral tegmental area activity in a reconstituted mesolimbic dopamine pathway. Brain Res Bull 120:159-165.

Berton O, McClung CA, Dileone RJ, Krishnan V, Renthal W, Russo SJ, Graham D, Tsankova NM, Bolanos CA, Rios M, Monteggia LM, Self DW, Nestler EJ (2006) Essential role of BDNF in the mesolimbic dopamine pathway in social defeat stress. Science 311:864-868.

Bittar TP, Nair BB, Kim JS, Chandrasekera D, Sherrington A, Iremonger KJ (2019) Corticosterone mediated functional and structural plasticity in corticotropin-releasing hormone neurons. Neuropharmacology 154: 79-86.

Bloomfield MA, McCutcheon RA, Kempton M, Freeman TP, Howes O (2019) The effects of psychosocial stress on dopaminergic function and the acute stress response. eLife 8:e46797.

Branco T, Staras K (2009) The probability of neurotransmitter release: variability and feedback control at single synapses. Nat Rev Neurosci 10:373383.

Broersen LM, Abbate F, Feenstra MG, de Bruin JP, Heinsbroek RP, Olivier B (2000) Prefrontal dopamine is directly involved in the anxiogenic interoceptive cue of pentylenetetrazol but not in the interoceptive cue of chlordiazepoxide in the rat. Psychopharmacology (Berl) 149:366-376.

Busquets-Garcia A, Bains J, Marsicano G (2018) CB1 receptor signaling in the brain: extracting specificity from ubiquity. Neuropsychopharmacology 43: $4-20$.

Cho K, Little HJ (1999) Effects of corticosterone on excitatory amino acid responses in dopamine-sensitive neurons in the ventral tegmental area. Neuroscience 88:837-845.

Coluccia D, Wolf OT, Kollias S, Roozendaal B, Forster A, de Quervain DJ (2008) Glucocorticoid therapy-induced memory deficits: acute versus chronic effects. J Neurosci 28:3474-3478.

Cottone P, Wang X, Park JW, Valenza M, Blasio A, Kwak J, Iyer MR, Steardo L, Rice KC, Hayashi T, Sabino V (2012) Antagonism of sigma-1 receptors blocks compulsive-like eating. Neuropsychopharmacology 37:2593-2604.

Daftary SS, Panksepp J, Dong Y, Saal DB (2009) Stress-induced, glucocorticoid-dependent strengthening of glutamatergic synaptic transmission in midbrain dopamine neurons. Neurosci Lett 452:273-276.

de Jong JW, Roelofs TJ, Mol FM, Hillen AE, Meijboom KE, Luijendijk MC, van der Eerden HA, Garner KM, Vanderschuren LJ, Adan RA (2015) Reducing ventral tegmental dopamine D2 receptor expression selectively boosts incentive motivation. Neuropsychopharmacology 40:2085-2095.

Di S, Malcher-Lopes R, Halmos KC, Tasker JG (2003) Nongenomic glucocorticoid inhibition via endocannabinoid release in the hypothalamus: a fast feedback mechanism. J Neurosci 23:4850-4857.

Di S, Itoga CA, Fisher MO, Solomonow J, Roltsch EA, Gilpin NW, Tasker JG (2016) Acute stress suppresses synaptic inhibition and increases anxiety via endocannabinoid release in the basolateral amygdala. J Neurosci 36:8461-8470.

Godfrey N, Borgland SL (2020) Sex differences in the effect of acute fasting on excitatory and inhibitory synapses onto ventral tegmental area dopamine neurons. J Physiol 598:5523-5539.

Gourley SL, Kiraly DD, Howell JL, Olausson P, Taylor JR (2008) Acute hippocampal brain-derived neurotrophic factor restores motivational and forced swim performance after corticosterone. Biol Psychiatry 64:884890.

Graf EN, Wheeler RA, Baker DA, Ebben AL, Hill JE, McReynolds JR, Robble MA, Vranjkovic O, Wheeler DS, Mantsch JR, Gasser PJ (2013) Corticosterone acts in the nucleus accumbens to enhance dopamine signaling and potentiate reinstatement of cocaine seeking. J Neurosci 33:11800-11810.

Harfstrand A, Fuxe K, Cintra A, Agnati LF, Zini I, Wikstrom AC, Okret S, Yu ZY, Goldstein M, Steinbusch H (1986) Glucocorticoid receptor immunoreactivity in monoaminergic neurons of rat brain. Proc Natl Acad Sci USA 83:9779-9783.

Heath CJ, Bussey TJ, Saksida LM (2015) Motivational assessment of mice using the touchscreen operant testing system: effects of dopaminergic drugs. Psychopharmacology (Berl) 232:4043-4057.

Hensleigh E, Pritchard LM (2013) Glucocorticoid receptor expression and sub-cellular localization in dopamine neurons of the rat midbrain. Neurosci Lett 556:191-195.

Hollon NG, Burgeno LM, Phillips PE (2015) Stress effects on the neural substrates of motivated behavior. Nat Neurosci 18:1405-1412.

Holroyd KB, Adrover MF, Fuino RL, Bock R, Kaplan AR, Gremel CM, Rubinstein M, Alvarez VA (2015) Loss of feedback inhibition via D2 autoreceptors enhances acquisition of cocaine taking and reactivity to drug-paired cues. Neuropsychopharmacology 40:1495-1509.

Jeziorski M, White FJ (1989) Dopamine agonists at repeated "autoreceptorselective" doses: effects upon the sensitivity of A10 dopamine autoreceptors. Synapse 4:267-280.

Joels M (2018) Corticosteroids and the brain. J Endocrinol 238:R121-R130.

Karatsoreos IN, Bhagat SM, Bowles NP, Weil ZM, Pfaff DW, McEwen BS (2010) Endocrine and physiological changes in response to chronic corticosterone: a potential model of the metabolic syndrome in mouse. Endocrinology 151:2117-2127.

Karst H, Joels M (2005) Corticosterone slowly enhances miniature excitatory postsynaptic current amplitude in mice CA1 hippocampal cells. J Neurophysiol 94:3479-3486.

Kim H, Yi JH, Choi K, Hong S, Shin KS, Kang SJ (2014) Regional differences in acute corticosterone-induced dendritic remodeling in the rat brain and their behavioral consequences. BMC Neurosci 15:65.

Kinlein SA, Shahanoor Z, Romeo RD, Karatsoreos IN (2017) Chronic corticosterone treatment during adolescence has significant effects on metabolism and skeletal development in male C57BL6/N mice. Endocrinology 158:2239-2254.

Kleen JK, Sitomer MT, Killeen PR, Conrad CD (2006) Chronic stress impairs spatial memory and motivation for reward without disrupting motor ability and motivation to explore. Behav Neurosci 120:842-851.

Lammel S, Ion DI, Roeper J, Malenka RC (2011) Projection-specific modulation of dopamine neuron synapses by aversive and rewarding stimuli. Neuron 70:855-862.

Liu S, Globa AK, Mills F, Naef L, Qiao M, Bamji SX, Borgland SL (2016) Consumption of palatable food primes food approach behavior by rapidly increasing synaptic density in the VTA. Proc Natl Acad Sci USA 113:2520-2525.

McEwen BS, De Kloet ER, Rostene W (1986) Adrenal steroid receptors and actions in the nervous system. Physiol Rev 66:1121-1188.

Mebel DM, Wong JC, Dong YJ, Borgland SL (2012) Insulin in the ventral tegmental area reduces hedonic feeding and suppresses dopamine concentration via increased reuptake. Eur J Neurosci 36:2336-2346.

Mehta MA, Gore-Langton E, Golembo N, Colvert E, Williams SC, SonugaBarke E (2010) Hyporesponsive reward anticipation in the basal ganglia following severe institutional deprivation early in life. J Cogn Neurosci 22:2316-2325.

Mitra R, Sapolsky RM (2008) Acute corticosterone treatment is sufficient to induce anxiety and amygdaloid dendritic hypertrophy. Proc Natl Acad Sci USA 105:5573-5578.

Moda-Sava RN, Murdock MH, Parekh PK, Fetcho RN, Huang BS, Huynh TN, Witztum J, Shaver DC, Rosenthal DL, Alway EJ, Lopez K, Meng Y, Nellissen L, Grosenick L, Milner TA, Deisseroth K, Bito H, Kasai H, Liston C (2019) Sustained rescue of prefrontal circuit dysfunction by antidepressant-induced spine formation. Science 364:eaat8078.

Myers B, McKlveen JM, Herman JP (2014) Glucocorticoid actions on synapses, circuits, and behavior: implications for the energetics of stress. Front Neuroendocrinol 35:180-196. 
Nasehi M, Mafi F, Oryan S, Nasri S, Zarrindast MR (2011) The effects of dopaminergic drugs in the dorsal hippocampus of mice in the nicotineinduced anxiogenic-like response. Pharmacol Biochem Behav 98:468473.

Pan B, Hillard CJ, Liu QS (2008) D2 dopamine receptor activation facilitates endocannabinoid-mediated long-term synaptic depression of GABAergic synaptic transmission in midbrain dopamine neurons via cAMP-protein kinase A signaling. J Neurosci 28:14018-14030.

Pickel VM, Chan J, Nirenberg MJ (2002) Region-specific targeting of dopamine D2-receptors and somatodendritic vesicular monoamine transporter 2 (VMAT2) within ventral tegmental area subdivisions. Synapse 45:113-124.

Pivonello R, Simeoli C, De Martino MC, Cozzolino A, De Leo M, Iacuaniello D, Pivonello C, Negri M, Pellecchia MT, Iasevoli F, Colao A (2015) Neuropsychiatric disorders in Cushing's syndrome. Front Neurosci 9:129.

Polter AM, Kauer JA (2014) Stress and VTA synapses: implications for addiction and depression. Eur J Neurosci 39:1179-1188.

Rhen T, Cidlowski JA (2005) Antiinflammatory action of glucocorticoids: new mechanisms for old drugs. N Engl J Med 353:1711-1723.

Ronken E, Mulder AH, Schoffelmeer AN (1994) Glucocorticoid and mineralocorticoid receptors differentially modulate cultured dopaminergic neurons of rat ventral mesencephalon. Eur J Pharmacol 263:149-156.

Russo SJ, Nestler EJ (2013) The brain reward circuitry in mood disorders. Nat Rev Neurosci 14:609-625.

Saal D, Dong Y, Bonci A, Malenka RC (2003) Drugs of abuse and stress trigger a common synaptic adaptation in dopamine neurons. Neuron 37:577-582.

Sakamoto K, Matsumura S, Okafuji Y, Eguchi A, Yoneda T, Mizushige T, Tsuzuki S, Inoue K, Fushiki T (2015) The opioid system contributes to the acquisition of reinforcement for dietary fat but is not required for its maintenance. Physiol Behav 138:227-235.

Sesack SR, Aoki C, Pickel VM (1994) Ultrastructural localization of D2 receptor-like immunoreactivity in midbrain dopamine neurons and their striatal targets. J Neurosci 14:88-106.

Sonino N, Fava GA (2001) Psychiatric disorders associated with Cushing's syndrome: epidemiology, pathophysiology and treatment. CNS Drugs 15:361-373.

Tasker JG, Herman JP (2011) Mechanisms of rapid glucocorticoid feedback inhibition of the hypothalamic-pituitary-adrenal axis. Stress 14:398-406.

Teegarden SL, Bale TL (2007) Decreases in dietary preference produce increased emotionality and risk for dietary relapse. Biol Psychiatry 61:1021-1029.

Tidey JW, Miczek KA (1996) Social defeat stress selectively alters mesocorticolimbic dopamine release: an in vivo microdialysis study. Brain Res 721:140-149.
Tournier BB, Steimer T, Millet P, Moulin-Sallanon M, Vallet P, Ibañez V, Ginovart N (2013) Innately low D2 receptor availability is associated with high novelty-seeking and enhanced behavioural sensitization to amphetamine. Int J Neuropsychopharmacol 16:1819-1834.

Tsai HC, Zhang F, Adamantidis A, Stuber GD, Bonci A, de Lecea L, Deisseroth K (2009) Phasic firing in dopaminergic neurons is sufficient for behavioral conditioning. Science 324:1080-1084.

Wagenmakers MA, Netea-Maier RT, Prins JB, Dekkers T, den Heijer M, Hermus AR (2012) Impaired quality of life in patients in long-term remission of Cushing's syndrome of both adrenal and pituitary origin: a remaining effect of long-standing hypercortisolism? Eur J Endocrinol 167:687-695.

Wall PM, Blanchard RJ, Markham C, Yang M, Blanchard DC (2004) Infralimbic D1 receptor agonist effects on spontaneous novelty exploration and anxiety-like defensive responding in CD-1 mice. Behav Brain Res 152:67-79.

Wanat MJ, Hopf FW, Stuber GD, Phillips PE, Bonci A (2008) Corticotropinreleasing factor increases mouse ventral tegmental area dopamine neuron firing through a protein kinase C-dependent enhancement of Ih. J Physiol 586:2157-2170.

Wheeler DS, Ebben AL, Kurtoglu B, Lovell ME, Bohn AT, Jasek IA, Baker DA, Mantsch JR, Gasser PJ, Wheeler RA (2017) Corticosterone regulates both naturally occurring and cocaine-induced dopamine signaling by selectively decreasing dopamine uptake. Eur J Neurosci 46:2638-2646.

Yasir M, Sonthalia S (2019) Corticosteroid adverse effects. StatPearls. Treasure Island, FL.

Young AM (2004) Increased extracellular dopamine in nucleus accumbens in response to unconditioned and conditioned aversive stimuli: studies using 1 min microdialysis in rats. J Neurosci Methods 138:57-63.

Zald DH, Cowan RL, Riccardi P, Baldwin RM, Ansari MS, Li R, Shelby ES, Smith CE, McHugo M, Kessler RM (2008) Midbrain dopamine receptor availability is inversely associated with novelty-seeking traits in humans. J Neurosci 28:14372-14378.

Zarrindast MR, Naghdi-Sedeh N, Nasehi M, Sahraei H, Bahrami F, Asadi F (2010) The effects of dopaminergic drugs in the ventral hippocampus of rats in the nicotine-induced anxiogenic-like response. Neurosci Lett 475:156-160.

Zweifel LS, Parker JG, Lobb CJ, Rainwater A, Wall VZ, Fadok JP, Darvas M, Kim MJ, Mizumori SJ, Paladini CA, Phillips PE, Palmiter RD (2009) Disruption of NMDAR-dependent burst firing by dopamine neurons provides selective assessment of phasic dopamine-dependent behavior. Proc Natl Acad Sci USA 106:7281-7288.

Zweifel LS, Fadok JP, Argilli E, Garelick MG, Jones GL, Dickerson TM, Allen JM, Mizumori SJ, Bonci A, Palmiter RD (2011) Activation of dopamine neurons is critical for aversive conditioning and prevention of generalized anxiety. Nat Neurosci 14:620-626. 